RIMS-1106, DTP-96-41

\title{
Boundary ABF Models
}

\author{
Tetsuji Miwa ${ }^{1}$ and Robert Weston ${ }^{2}$
}

October 1996

\begin{abstract}
We diagonalise the transfer matrix of boundary ABF models using bosonized vertex operators. We compute the boundary S-matrix and check the scaling limit against known results for perturbed boundary conformal field theories.
\end{abstract}

\section{Introduction}

The theory of solvable lattice models, which originated in the work of Bethe and Onsager in the 3040 's, has matured in the last 20 years. Various models were found to be solvable and various methods were invented to solve these models. In the course of this work, some remarkable interactions occured between the theory of solvable lattice models and other branches of mathematics and physics, e.g., representation theory and conformal field theory.

The ABF models we are going to study in this paper were introduced in [1]. Baxter's book [2] presented the corner transfer matrix method, by which the one point correlation functions were computed. The general results obtained in [1] strongly suggested a link between solvable lattice models and the representation theory of the Virasoro algebra.

Such a link was manifest from the very beginning in conformal field theory. The representation theory of the Virasoro algebra was very successfully applied to conformal field theory to obtain correlation functions and the differential equations which they obeyed.

Solvability of lattice models was understood by means of the method of commuting transfer matrices. The algebraic structure behind it was given shape by the idea of $q$-deformation. Finally, in [3, 4], the corner transfer matrix method was given its correct place in the representation theory. The link was established between the highest weight representations of the quantum affine algebra $U_{q}\left(\widehat{s l}_{2}\right)$ and the XXZ model in the anti-ferromagnetic regime. Namely, the spaces of the eigenvectors of the corner transfer matrix of the latter were identified with the level 1 highest weight representations of the former, and the half transfer matrices were identified with the intertwiners of these representations. In fact, a similar structure had been known in conformal field theory. The operators of this kind were called vertex operators. These operators were realized using representation theory [5]. The method of bosonization was also very powerful [6].

\footnotetext{
${ }^{1}$ Research Institute for Mathematical Sciences, Kyoto University, Kyoto 606, Japan.

${ }^{2}$ Department of Mathematical Sciences, University of Durham, Science Labs, South Rd, Durham DH1 3LE, U.K. R.A.Weston@durham.ac.uk
} 
As for the ABF models, such a scheme was only recently used successfully [7]. The bosonization method of conformal field theory, by which the minimal unitary models were solved, was deformed to give a realization of the half transfer matrices of the $\mathrm{ABF}$ models.

The boundary problem has been studied in both lattice and continuum theories. In [8], the vertex operator method was extended to the boundary XXZ model. The aim of this paper is to extend the bosonization method to the boundary ABF models.

We start from a known solution of the boundary Yang-Baxter equation [9]. We then introduce the boundary transfer matrix of Sklyanin-type in terms of a product of vertex operators. Our goal is to diagonalise the boundary transfer matrix. We shall give an explicit formula for the boundary vacuum state and compute the boundary $S$-matrix. The bosonization scheme of [f] is based on a cohomological construction. Thus, in the boundary ABF models, the physical spaces of states are realized as sub-quotients of the bosonic Fock spaces on which the screening operators act. One of the main points in our work is to show that the boundary vacuum states belong to the sub-quotients.

The scaling limit of the bulk ABF models in regime III is described by the $\phi_{(1,3)}$ deformations of the $c=1-6 / r(r-1)$ rational conformal field theories. In [10] and [11], boundary S-matrices are computed for the perturbed $r=4$ and $r=5$ models on a half line (the Ising and Tri-critical Ising models respectively). We check that our boundary S-matrices agree with these results in the scaling limit.

The plan of the paper is as follows. Section 2 prepares the boundary Boltzmann weights and the transfer matrix. Section 3 is a summary of the bosonization scheme. We give the boundary vacuum state in Section 4, and compute the boundary $S$-matrices in Section 5 . Section 6 takes the scaling limit. Appendix A gives useful formulas for operator products. Appendix B gives a proof of the eigenvector relation for the boundary vacuum states.

\section{Bulk and boundary weights}

We recall the Boltzmann weights of the $\mathrm{ABF}$ model, and set up the boundary transfer matrix in terms of the vertex operators.

\subsection{The bulk weights}

The ABF model has two parameters $x$ and $r$. We assume that $0<x<1$ and $r \geqslant 4(r \in \mathbb{Z})$. We use the symbol $[u]$ for the elliptic theta function.

$$
\begin{aligned}
& {[u]=x^{\frac{u^{2}}{r}-u} \Theta_{x^{2 r}}\left(x^{2 u}\right),} \\
& \Theta_{q}(z)=(z ; q)_{\infty}\left(q z^{-1} ; q\right)_{\infty}(q ; q)_{\infty} \\
& \left(z ; q_{1}, \cdots, q_{m}\right)_{\infty}=\prod_{i_{1}, \cdots, i_{m}=0}^{\infty}\left(1-q_{1}^{i_{1}} \cdots q_{m}^{i_{m}} z\right) .
\end{aligned}
$$


There are six kinds of configurations around a face and the corresponding Boltzmann weights are given as follows [1].

$$
\begin{aligned}
& W\left(\begin{array}{cc|}
k \pm 1 & k \\
k & k \mp 1
\end{array} \mid u\right)=R(u) \\
& W\left(\begin{array}{cc|c}
k & k \pm 1 \\
k \pm 1 & k & u
\end{array}\right)=R(u) \frac{[k \pm u][1]}{[1-u][k]} \\
& W\left(\begin{array}{cc|c}
k & k \pm 1 \\
k \mp 1 & k & u
\end{array}\right)=R(u) \frac{[k \mp 1][u]}{[k][1-u]}
\end{aligned}
$$

Here $k$ is an integer such that $1 \leqslant k \leqslant r-1$, and the normalisation factor $R(u)$ is given by

$$
\begin{aligned}
R(u) & =\zeta^{\frac{r-1}{2 r}} \frac{\rho(\zeta)}{\rho\left(\zeta^{-1}\right)}, \quad \zeta=x^{2 u} \\
\rho(\zeta) & =\frac{\left(x^{4} \zeta ; x^{4}, x^{2 r}\right)_{\infty}\left(x^{2 r} \zeta ; x^{4}, x^{2 r}\right)_{\infty}}{\left(x^{2} \zeta ; x^{4}, x^{2 r}\right)_{\infty}\left(x^{2+2 r} \zeta ; x^{4}, x^{2 r}\right)_{\infty}} .
\end{aligned}
$$

Graphically, we represent $W\left(\begin{array}{cc}d & c \\ a & b\end{array} \mid u\right)$ by

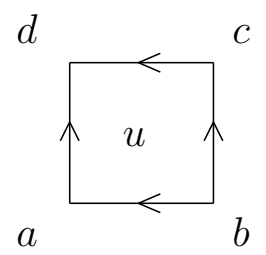

The Boltzmann weights satisfy the following relations.

- Yang-Baxter equation

$$
\begin{aligned}
& \sum_{g} W\left(\begin{array}{ll}
f & g \\
a & b
\end{array} \mid u-v\right) W\left(\begin{array}{ll}
g & d \\
b & c
\end{array} \mid u\right) W\left(\begin{array}{ll}
f & e \\
g & d
\end{array} \mid v\right) \\
= & \sum_{g} W\left(\begin{array}{ll}
a & g \\
b & c
\end{array} \mid v\right) W\left(\begin{array}{ll}
f & e \\
a & g
\end{array} \mid u\right) W\left(\begin{array}{ll}
e & d \\
g & c
\end{array} \mid u-v\right) .
\end{aligned}
$$

- Unitarity relation

$$
\sum_{g} W\left(\begin{array}{ll}
d & g \\
a & b
\end{array} \mid u\right) W\left(\begin{array}{ll}
d & c \\
g & b
\end{array} \mid-u\right)=\delta_{a c} .
$$

- Crossing relation

$$
W\left(\begin{array}{ll}
d & c \\
a & b
\end{array} \mid u\right)=\frac{[a]}{[b]} W\left(\begin{array}{cc|}
a & d \\
b & c
\end{array} \mid 1-u\right)
$$




\subsection{The vertex operators}

Consider the corner transfer matrices $A(\zeta), B(\zeta), C(\zeta), D(\zeta)$ which represent the $N W, S W, S E, N E$ quadrants, respectively. Let $\mathcal{L}_{l, k}$ be the space of the eigenvectors of $A(\zeta)$ in the sector such that the central height is equal to $k$ and the boundary heights are $(l, l+1)$. We denote by $\Phi^{(k+\varepsilon, k)}(\zeta)$ the half-infinite transfer matrix extending to infinity in the north. This is an operator

$$
\Phi^{(k+\varepsilon, k)}(\zeta): \mathcal{L}_{l, k} \longrightarrow \mathcal{L}_{l, k+\varepsilon} .
$$

Similarly, we denote by $\Phi^{*(k, k+\varepsilon)}\left(\zeta^{-1}\right)$ the half-infinite transfer matrix extending to infinity in the west. This is an operator

$$
\Phi^{*(k, k+\varepsilon)}\left(\zeta^{-1}\right): \mathcal{L}_{l, k+\varepsilon} \longrightarrow \mathcal{L}_{l, k}
$$

They satisfy the following relations.

- Exchange relation

$$
\sum_{g} W\left(\begin{array}{cc}
a & g \\
b & c
\end{array} \mid u_{2}-u_{1}\right) \Phi^{(a, g)}\left(\zeta_{2}\right) \Phi^{(g, c)}\left(\zeta_{1}\right)=\Phi^{(a, b)}\left(\zeta_{1}\right) \Phi^{(b, c)}\left(\zeta_{2}\right) .
$$

- Duality

$$
\Phi^{*(k, k+\varepsilon)}(\zeta)=\frac{[1]}{[k]} \Phi^{(k, k+\varepsilon)}\left(x^{2} \zeta\right)
$$

- Inversion relation

$$
\sum_{g} \Phi^{*(a, g)}(\zeta) \Phi^{(g, a)}(\zeta)=1, \quad \Phi^{(a, b)}(\zeta) \Phi^{*(b, c)}(\zeta)=\delta_{a c}
$$

These operators are realized as vertex operators acting on the bosonic Fock space $\mathcal{F}_{l, k}$ in Section 3.1.

\subsection{The boundary weights.}

We follow Sklyanin's scheme in dealing with the boundary ABF model [12]. Boundary weights

$$
K\left(\begin{array}{c}
k+\varepsilon \\
k+\varepsilon^{\prime}
\end{array}\right) \quad\left(\varepsilon, \varepsilon^{\prime}= \pm\right)
$$

are given to the boundary configurations. We restricts to the diagonal case, $\varepsilon=\varepsilon^{\prime}$, and use a solution of the boundary Yang-Baxter equation,

$$
\begin{aligned}
& \sum_{f, g} W\left(\begin{array}{cc}
c & f \\
b & a
\end{array} \mid u-v\right) W\left(\begin{array}{cc}
c & d \\
f & g
\end{array} \mid u+v\right) K\left(\begin{array}{l}
g \\
a
\end{array} \mid u\right) K\left(\begin{array}{l}
e \\
g
\end{array} \mid v\right) \\
= & \sum_{f, g} W\left(\begin{array}{cc}
c & d \\
f & e
\end{array} \mid u-v\right) W\left(\begin{array}{ll}
c & f \\
b & g
\end{array} \mid u+v\right) K\left(\begin{array}{c}
e \\
g
\end{array} \mid u\right) K\left(\begin{array}{l}
g \\
a
\end{array} \mid v\right) .
\end{aligned}
$$


The solution is given by [9]

$$
\frac{K\left(\begin{array}{ll|}
k+1 & k \\
& k
\end{array} \mid u\right)}{K\left(\begin{array}{l}
k-1 \\
k
\end{array} \mid u\right)}=\frac{[c+u][k+c-u]}{[c-u][k+c+u]} .
$$

Here the constant $c$ is arbitrary. We choose to restrict $c$ to lie in one of two separate regions parameterised by A) $x^{2 c}=-x^{2 b},-1<b<1$; B) $x^{2 c}=x^{2 b},-1<b<1$. We further restrict $u$ to satisfy $0<u<|b|<1$ in both regions. The overall factor in $K\left(k \pm 1 \begin{array}{l}k \\ \end{array} \mid u\right)$ is determined later.

Graphically, we represent $K\left({ }_{c}^{b} \mid u\right)$ by

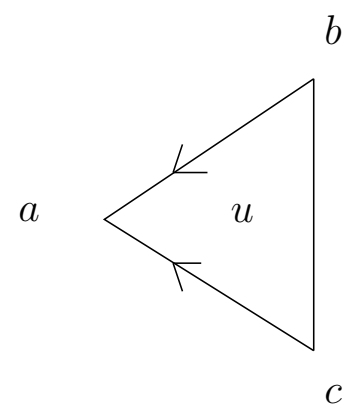

\subsection{The boundary transfer matrix.}

We define the boundary transfer matrix

$$
T_{B}^{(k)}(u)=\sum_{\varepsilon} \Phi^{*(k, k+\varepsilon)}\left(\zeta^{-1}\right) K\left(k+\varepsilon{ }_{k}^{k} \mid u\right) \Phi^{(k+\varepsilon, k)}(\zeta) .
$$

The boundary Yang-Baxter equation implies [12, 9]

$$
\left[T_{B}^{(k)}(u), T_{B}^{(k)}(v)\right]=0
$$

Graphically, $T_{B}^{(k)}(u)$ is represented by the following half-infinite transfer matrix

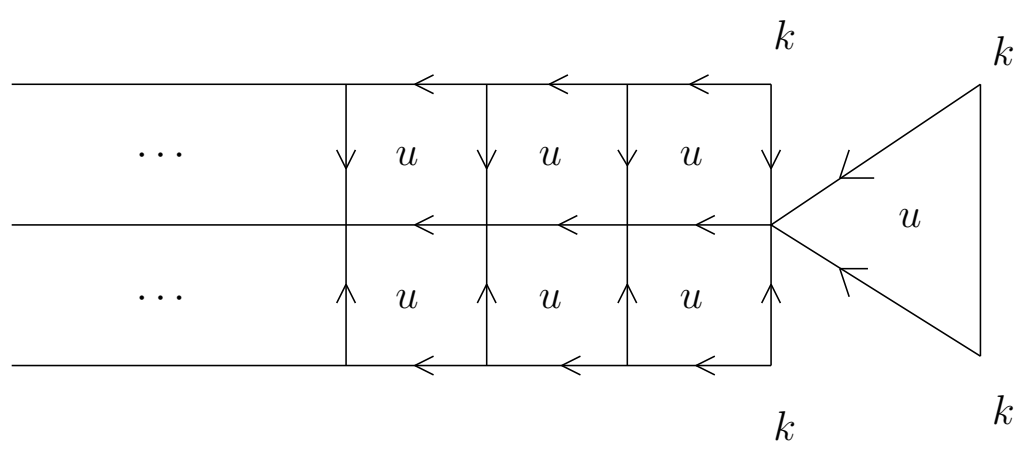


We also define the boundary Hamiltonian $H_{B}^{(k)}$ in terms of the transfer matrix as

$$
H_{B}^{(k)}=\left.\frac{\left(1-x^{2}\right)}{2 x} \frac{\zeta d T_{B}^{(k)}(\zeta)}{d \zeta}\right|_{\zeta=1} .
$$

Our first question is about the ground state configuration, i.e., the largest eigenvalue eigenvector of $T_{B}^{(k)}(u)$ in the limit $x \rightarrow 0$. We know that in the bulk theory the ground state configuration of heights is given by the alternating sequence of $l$ and $l+1$. Because we have chosen to fix the heights at the right-most corners to be equal to $k$, it follows that the possible choices are $l=k$ or $k-1$. Let us compare $K\left(k \pm 1 \quad \begin{array}{l}k \\ \end{array}\right.$

$$
\frac{K\left(k+1_{k}^{k} \mid u\right)}{K\left(k-r_{k}^{k} \mid u\right)}=\left\{\begin{array}{lll}
>1 & \text { if } & b>0 \\
<1 & \text { if } & b<0 .
\end{array}\right.
$$

This is true in both regions $\mathrm{A}$ and $\mathrm{B}$. Therefore, the ground state configuration for $b>0$ is given by $l=k$, and for $b<0$ it is given by $l=k-1$.

Next, let us fix the overall factor in the boundary weights. The normalisation will differ de-

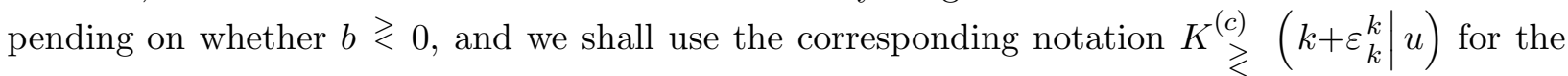
normalised boundary weights.

First, suppose $b>0$, and set

$$
\begin{aligned}
& \bar{K}_{>}^{(c)}\left(k+r_{k}^{k} \mid u\right)=1 \\
& \bar{K}_{>}^{(c)}\left(k-r_{k}^{k} \mid u\right)=\frac{[c-u][k+c+u]}{[c+u][k+c-u]} .
\end{aligned}
$$

The corresponding boundary transfer matrix $\bar{T}_{B}^{(k)}(u)$ satisfies

$$
\begin{aligned}
& \bar{T}_{B}^{(k)}(-u) \bar{T}_{B}^{(k)}(u)=1 \\
& \bar{T}_{B}^{(k)}(u)=\frac{[2-2 u][c+u][k+c-u]}{R(2 u-1)[2 u][1+c-u][k+c-1+u]} \bar{T}_{B}^{(k)}(1-u) .
\end{aligned}
$$

Since $\left\{\bar{T}_{B}^{(k)}(u)\right\}$ is a commuting family, the eigenvalues of $\bar{T}_{B}^{(k)}(u)$ also satisfy these equations. Assuming the analyticity of $\log \bar{t}_{B}^{(k)}(u)$ for the largest eigenvalue $\bar{t}_{B}^{(k)}(u)$ in a neighbourhood of the annulus $x \leq|\zeta| \leq 1$, we determine $\bar{t}_{B}^{(k)}(u)$. After that we choose the normalisation of $K_{>}^{(c)}\left(k \pm 1_{k}^{k} \mid u\right)$ in such a way that $T_{B}^{(k)}(u)$ has the largest eigenvalue 1 . The result is as follows

$$
\begin{aligned}
K_{>}^{(c)}\left(k+1_{k}^{k} \mid u\right) & =h_{>}^{(k)}(u) \\
K_{>}^{(c)}\left(k-1_{k}^{k} \mid u\right) & =h_{>}^{(k)}(u) \frac{[c-u][k+c+u]}{[c+u][k+c-u]},
\end{aligned}
$$




$$
\begin{aligned}
h_{>}^{(k)}(u) & =\zeta^{\frac{r-1-2 k}{2 r}} \frac{f(\zeta) p_{>}^{(k)}(\zeta) p_{>}^{(k)}\left(x^{2} \zeta^{-1}\right)}{f\left(\zeta^{-1}\right) p_{>}^{(k)}\left(\zeta^{-1}\right) p_{>}^{(k)}\left(x^{2} \zeta\right)}, \\
f(\zeta) & =\frac{\left(x^{2 r} \zeta^{2} ; x^{8}, x^{2 r}\right)_{\infty}\left(x^{8} \zeta^{2} ; x^{8}, x^{2 r}\right)_{\infty}}{\left(x^{6} \zeta^{2} ; x^{8}, x^{2 r}\right)_{\infty}\left(x^{2+2 r} \zeta^{2} ; x^{8}, x^{2 r}\right)_{\infty}}, \\
p_{>}^{(k)}(\zeta) & =\frac{\left(x^{2(1+c)} \zeta ; x^{4}, x^{2 r}\right)_{\infty}\left(x^{2(r-c-k+1)} \zeta ; x^{4}, x^{2 r}\right)_{\infty}}{\left(x^{2(r-c)} \zeta ; x^{4}, x^{2 r}\right)_{\infty}\left(x^{2(c+k)} \zeta ; x^{4}, x^{2 r}\right)_{\infty}} .
\end{aligned}
$$

This normalisation of $K_{>}^{(c)}\left(k \pm 1_{k}^{k} \mid u\right)$ is valid for both regions $A$ and $B$ when $b>0$. The case $b<0$ is similar. It is even not necessary to repeat the calculation because we have a symmetry $(c, k, \pm) \rightarrow(-c, r-k, \mp)$. Therefore, we have

$$
\begin{gathered}
K_{<}^{(c)}\left(k+1 \frac{k}{k} \mid u\right)=h_{<}^{(k)}(u) \frac{[c+u][k+c-u]}{[c-u][k+c+u]}, \\
K_{<}^{(c)}\left(k-1 \frac{k}{k} \mid u\right)=h_{<}^{(k)}(u), \\
h_{<}^{(k)}(u)=\zeta^{\frac{2 k-1-r}{2 r}} \frac{f(\zeta) p_{<}^{(k)}(\zeta) p_{<}^{(k)}\left(x^{2} \zeta^{-1}\right)}{f\left(\zeta^{-1}\right) p_{<}^{(k)}\left(\zeta^{-1}\right) p_{<}^{(k)}\left(x^{2} \zeta\right)}, \\
p_{<}^{(k)}(\zeta)=\frac{\left(x^{2(1-c)} \zeta ; x^{4}, x^{2 r}\right)_{\infty}\left(x^{2(c+k+1)} ; x^{4}, x^{2 r}\right)_{\infty}}{\left(x^{2(r+c)} \zeta ; x^{4}, x^{2 r}\right)_{\infty}\left(x^{2(r-c-k)} \zeta ; x^{4}, x^{2 r}\right)_{\infty}} .
\end{gathered}
$$

In conclusion, we have fixed the normalisation of the boundary weights for $b>0$ and $b<0$ separately so that the largest eigenvalue of the boundary transfer matrix is 1 .

For completeness, we give two relations for $T_{B}^{(k)}(u)$ and the related relations for $K_{\gtrless}^{(c)}\left(k+\varepsilon_{k}^{k} \mid u\right)$.

$$
\begin{aligned}
& T_{B}^{(k)}(-u) T_{B}^{(k)}(u)=1, \\
& T_{B}^{(k)}(1-u)=T_{B}^{(k)}(u), \\
& K^{(c)} \underset{\gtrless}{\gtrless}\left(\begin{array}{r}
k^{\prime} \\
k \\
k
\end{array} \mid u\right) K_{2}^{(c)} \gtrless\left(\begin{array}{r}
k^{\prime} \\
k
\end{array} \mid-u\right)=1, \\
& K_{\stackrel{(c)}{\gtrless}}^{\gtrless}\left(k^{\prime} \begin{array}{c}
k \\
k
\end{array} \mid 1-u\right)=\sum_{k^{\prime \prime}} W\left(\begin{array}{cc}
k & k^{\prime} \\
k^{\prime \prime} & k
\end{array} \mid 2 u-1\right) \frac{\left[k^{\prime \prime}\right]}{\left[k^{\prime}\right]} K_{\gtrless}^{(c)} \underset{\gtrless}{\gtrless}\left(\begin{array}{r}
k^{\prime \prime} k \\
k
\end{array} \mid u\right) .
\end{aligned}
$$

\section{Bosonization}

We follow [7] to obtain a bosonic realization of the spaces $\mathcal{L}_{l, k}$, their duals, and the vertex operators.

\subsection{Boson Fock spaces}

We use the bosonic oscillators

$$
\begin{aligned}
{\left[\beta_{m}, \beta_{n}\right] } & =m \frac{[m]_{x}[(r-1) m]_{x}}{[2 m]_{x}[r m]_{x}} \delta_{m+n, 0} \\
{[P, Q] } & =-i
\end{aligned}
$$


where $[k]_{x}=\frac{x^{k}-x^{-k}}{x-x^{-1}}$. The Fock spaces are

$$
\begin{aligned}
\mathcal{F}_{l, k} & =\mathbf{C}\left[\beta_{-1}, \beta_{-2}, \cdots\right]|l, k\rangle, \\
\mathcal{F}_{l, k}^{*} & =\langle l, k| \mathbf{C}\left[\beta_{1}, \beta_{2}, \cdots\right], \\
\langle l, k \mid l, k\rangle & =1
\end{aligned}
$$

where

$$
\begin{aligned}
& \beta_{m}|l, k\rangle=0 \text { if } m \geq 1, \\
& \langle l, k| \beta_{m}=0 \text { if } m \leq-1
\end{aligned}
$$

The operator $P$ acts as

$$
l \sqrt{\frac{r}{2(r-1)}}-k \sqrt{\frac{r-1}{2 r}} \text { on } \mathcal{F}_{l, k} \text { and } \mathcal{F}_{l, k}^{*} .
$$

We also use operators $K$ and $L$ which act on $\mathcal{F}_{l, k}, \mathcal{F}_{l, k}^{*}$ as $k$ and $l$, respectively. We use the convention of left action on $\mathcal{F}_{l, k}$ and right action on $\mathcal{F}_{l, k}^{*}$. Namely, the composition $A B$ of operators $A$ and $B$ is such that $B$ acts first on $\mathcal{F}_{l, k}$ and $A$ acts first on $\mathcal{F}_{l, k}^{*}$.

\subsection{Type I and II vertex operators}

We define the type I vertex operators

$$
\begin{aligned}
\Phi_{+}(\zeta) & =\zeta^{-\frac{r-1}{4 r}}: e^{-i \sqrt{\frac{r-1}{2 r}}(Q+i P \log \zeta)-\sum_{m \neq 0} \frac{\beta_{m}}{m} \zeta^{m}}: \\
\Phi_{-}(\zeta) & =\lambda^{-1} \oint_{C} \frac{d z}{2 \pi i z} \Phi_{+}(\zeta) A(z) \frac{\left[u+v+\frac{1}{2}-K\right]}{\left[u+v-\frac{1}{2}\right]} \\
A(z) & =z^{\frac{r-1}{r}}: e^{i \sqrt{\frac{2(r-1)}{r}}(Q-i P \log z)+\sum_{m \neq 0} \frac{\beta_{m}}{m}\left(x^{m}+x^{-m}\right) z^{-m}}:
\end{aligned}
$$

where

$$
\begin{aligned}
& \zeta=x^{2 u}, \quad z=x^{2 v}, \\
& \lambda=x^{\frac{1-r}{2 r}}\left(x^{2 r-2} ; x^{2 r}\right)_{\infty} \frac{\left(x^{4} ; x^{4}, x^{2 r}\right)_{\infty}\left(x^{4+2 r} ; x^{4}, x^{2 r}\right)_{\infty}}{\left(x^{2} ; x^{4}, x^{2 r}\right)_{\infty}\left(x^{2+2 r} ; x^{4}, x^{2 r}\right)_{\infty}}
\end{aligned}
$$

The contour $C$ for the $z$-integration in $\Phi_{-}(\zeta)$ is chosen in such a way that the poles from the factor $\left[u+v-\frac{1}{2}\right]$ at $z=x^{1+2 n r} / \zeta$ are inside if $n \in \mathbb{Z}_{\geq 0}$ (outside if $n \in \mathbb{Z}_{<0}$ )), and the poles from the normal ordering of the product $\Phi_{+}(\zeta) A(z)$ at $z=1 / x^{1+2 n r} \zeta\left(n \in \mathbb{Z}_{\geq 0}\right)$ are outside. The operator $\Phi_{ \pm}(\zeta)$ acts as

$$
\begin{array}{ll}
\Phi_{ \pm}(\zeta) & : \quad \mathcal{F}_{l, k} \rightarrow \mathcal{F}_{l, k \pm 1}, \\
\Phi_{ \pm}(\zeta) & : \quad \mathcal{F}_{l, k \pm 1}^{*} \rightarrow \mathcal{F}_{l, k}^{*} .
\end{array}
$$

The value of $K$ in $\Phi_{-}(\zeta)$ is $k$ in both cases. 
The type II vertex operators are

$$
\begin{aligned}
& \Psi_{+}^{*}(\zeta)=\zeta^{-\frac{r}{4(r-1)}}: e^{i \sqrt{\frac{r}{2(r-1)}}(Q+i P \log \zeta)+\sum_{m \neq 0} \frac{\alpha_{m}}{m} \zeta^{m}}:, \\
& \Psi_{-}^{*}(\zeta)=\oint_{C^{\prime}} \frac{d z}{2 \pi i z} \Psi_{+}^{*}(\zeta) B(z) \frac{\left[\frac{1}{2}-L-u-v\right]^{\prime}}{\left[u+v+\frac{1}{2}\right]^{\prime}},
\end{aligned}
$$

where

$$
\begin{aligned}
\alpha_{m} & =(-1)^{m} \frac{[r m]_{x}}{[(r-1) m]_{x}} \beta_{m}, \\
B(z) & =z^{\frac{r}{r-1}}: e^{-i \sqrt{\frac{2 r}{r-1}}(Q-i P \log z)-\sum_{m \neq 0} \frac{\alpha_{m}}{m}\left(x^{m}+x^{-m}\right) z^{-m}}: \\
{[u]^{\prime} } & =x^{\frac{u^{2}}{r-1}-u} \Theta_{x^{2(r-1)}}\left(x^{2 u}\right) .
\end{aligned}
$$

The contour $C^{\prime}$ for the $z$-integration in $\Psi_{-}^{*}(\zeta)$ is chosen in such a way that the poles from the factor $\left[u+v+\frac{1}{2}\right]^{\prime}$ at $z=x^{2 n(r-1)-1} / \zeta$ are inside if $n \in \mathbb{Z}_{\geq 0}$ (outside if $n \in \mathbb{Z}_{<0}$ ) and the poles from the normal ordering of the product $\Psi_{+}^{*}(\zeta) B(z)$ at $z=x^{1-2 n(r-1)} / \zeta\left(n \in \mathbb{Z}_{\geq 0}\right)$ are outside. The operator $\Psi_{ \pm}^{*}(\zeta)$ acts as

$$
\begin{aligned}
& \Psi_{ \pm}^{*}(\zeta): \mathcal{F}_{l, k} \rightarrow \mathcal{F}_{l \pm 1, k}, \\
& \Psi_{ \pm}^{*}(\zeta): \mathcal{F}_{l \pm 1, k}^{*} \rightarrow \mathcal{F}_{l, k}^{*} \text {. }
\end{aligned}
$$

The value of $L$ in $\Psi_{-}^{*}(\zeta)$ is $l$ in both cases.

The vertex operators satisfy the following commutation relations.

$$
\begin{aligned}
& \Phi_{\varepsilon_{2}}\left(\zeta_{2}\right) \Phi_{\varepsilon_{1}}\left(\zeta_{1}\right) \\
& =\sum_{\varepsilon_{1}^{\prime}+\varepsilon_{2}^{\prime}=\varepsilon_{1}+\varepsilon_{2}} W\left(\begin{array}{cc}
K+\varepsilon_{1}+\varepsilon_{2} & K+\varepsilon_{2}^{\prime} \\
K+\varepsilon_{1} & K
\end{array} \mid u_{1}-u_{2}\right) \Phi_{\varepsilon_{1}^{\prime}}\left(\zeta_{1}\right) \Phi_{\varepsilon_{2}^{\prime}}\left(\zeta_{2}\right), \\
& \quad \sum_{\varepsilon} \Phi_{\varepsilon}^{*}(\zeta) \Phi_{\varepsilon}(\zeta)=1 \\
& \Phi_{\varepsilon^{\prime}}(\zeta) \Phi_{\varepsilon}^{*}(\zeta)=\delta_{\varepsilon^{\prime} \varepsilon}
\end{aligned}
$$

where

$$
\Phi_{\varepsilon}^{*}(\zeta)=\frac{[1]}{[K]} \Phi_{-\varepsilon}\left(x^{2} \zeta\right)
$$

Comparing (3.49)-(3.51) with (2.7)-(2.9), we see that $\Phi_{\varepsilon}(\zeta)$ and $\Phi_{\varepsilon}^{*}\left(\zeta^{-1}\right)$ provide a Fock space realisation of the half-infinite transfer matrices $\Phi^{(k+\varepsilon, k)}(\zeta)$ and $\Phi^{*(k, k+\varepsilon)}\left(\zeta^{-1}\right)$ respectively.

We also have

$$
\Psi_{\varepsilon_{2}}^{*}\left(\zeta_{2}\right) \Psi_{\varepsilon_{1}}^{*}\left(\zeta_{1}\right)
$$




$$
\begin{aligned}
& =-\sum_{\varepsilon_{1}^{\prime}+\varepsilon_{2}^{\prime}=\varepsilon_{1}+\varepsilon_{2}} W^{\prime}\left(\begin{array}{cc}
L+\varepsilon_{1}+\varepsilon_{2} & L+\varepsilon_{2}^{\prime} \\
L+\varepsilon_{1} & L
\end{array} \mid u_{2}-u_{1}\right) \Psi_{\varepsilon_{1}^{\prime}}^{*}\left(\zeta_{1}\right) \Psi_{\varepsilon_{2}^{\prime}}^{*}\left(\zeta_{2}\right), \\
& \Phi_{\varepsilon_{1}}\left(\zeta_{1}\right) \Psi_{\varepsilon_{2}}^{*}\left(\zeta_{2}\right)=\chi\left(\zeta_{2} / \zeta_{1}\right) \Psi_{\varepsilon_{2}}^{*}\left(\zeta_{2}\right) \Phi_{\varepsilon_{1}}\left(\zeta_{1}\right),
\end{aligned}
$$

where $W^{\prime}\left(\begin{array}{ll}d & c \\ a & b\end{array} \mid u\right)$ is the Boltzmann weight with $r$ replaced by $r-1$, and

$$
\chi(\zeta)=\zeta^{-1 / 2} \frac{\Theta_{x^{4}}(-x \zeta)}{\Theta_{x^{4}}\left(-x \zeta^{-1}\right)}
$$

Set

$$
\Psi_{\varepsilon}(\zeta)=\Psi_{-\varepsilon}^{*}\left(x^{2} \zeta\right) \frac{[1]^{\prime}}{[L]^{\prime}}
$$

We then have a pole

$$
\Psi_{\varepsilon}\left(\zeta_{1}\right) \Psi_{\varepsilon}^{*}\left(\zeta_{2}\right)=\frac{\varepsilon g}{1-\zeta_{1} / \zeta_{2}}+\cdots
$$

where

$$
g=x^{\frac{r}{2(r-1)}} \frac{\left(x^{2 r} ; x^{2(r-1)}\right)_{\infty}\left(x^{2} ; x^{4}, x^{2(r-1)}\right)_{\infty}\left(x^{2 r} ; x^{4}, x^{2(r-1)}\right)_{\infty}}{\left(x^{2(r-1)} ; x^{2(r-1)}\right)_{\infty}\left(x^{4} ; x^{4}, x^{2(r-1)}\right)_{\infty}\left(x^{2(r-1)} ; x^{4}, x^{2(r-1)}\right)_{\infty}} .
$$

Our bosonization is slightly different from than that of reference [7]. Firstly, we have changed $\zeta$ to $\zeta^{-1}$ in the definition of $\Phi_{ \pm}$. Secondly, our $\Phi_{-}$involves an extra factor of $(-1)^{K-L}$ over the corresponding object $\Psi^{-}$of [0]. This factor arises because our Boltzmann weights $W$ differ from $U$ of [7] by a - sign in (2.3). This difference is simply a gauge transformation. Finally, our $\alpha_{m}$ is equal to $(-1)^{m} \alpha_{m}$ of [7]. We introduce this factor in order to give the correct sign in the commutation relations (3.54).

\subsection{Screening operators}

We define two screening operators $X$ and $Y$ that have the properties [7, 13].

$$
\begin{aligned}
& X: \mathcal{F}_{l, k} \rightarrow \mathcal{F}_{l-2, k} \quad\left(\mathcal{F}_{l-2, k}^{*} \rightarrow \mathcal{F}_{l, k}^{*}\right) \\
& Y: \mathcal{F}_{l, k} \rightarrow \mathcal{F}_{l, k-2} \quad\left(\mathcal{F}_{l, k-2}^{*} \rightarrow \mathcal{F}_{l, k}^{*}\right) \\
& {\left[\Phi_{\varepsilon}(\zeta), X\right]=0, \quad\left[\Psi_{\varepsilon}^{*}(\zeta), Y\right]=0, \quad[X, Y]=0} \\
& X^{r-1}=0, \quad Y^{r}=0
\end{aligned}
$$

They have the following bosonization.

$$
\begin{aligned}
& X=\oint \frac{d z}{2 \pi i z} B(z) \frac{\left[\frac{1}{2}-L-v\right]^{\prime}}{\left[\frac{1}{2}+v\right]^{\prime}}, \\
& Y=\oint \frac{d z}{2 \pi i z} A(z) \frac{\left[\frac{1}{2}-K+v\right]}{\left[\frac{1}{2}-v\right]} .
\end{aligned}
$$


It is proved in [13] that these expressions obey (3.62).

Fix $l(1 \leq l \leq r-1)$ and $k(1 \leq l \leq r)$. We have the following complexes.

$$
\begin{gathered}
\ldots \stackrel{X_{-2}=X^{l}}{\longrightarrow} \mathcal{F}_{2(r-1)-l, k} \stackrel{X_{-1}=X^{r-1-l}}{\longrightarrow} \mathcal{F}_{l, k} \stackrel{X_{0}=X^{l}}{\longrightarrow} \mathcal{F}_{-l, k} \stackrel{X_{1}=X^{r-1-l}}{\longrightarrow} \cdots \\
\ldots \stackrel{X_{-2}^{*}=X^{l}}{\longleftarrow} \mathcal{F}_{2(r-1)-l, k}^{*} \stackrel{X_{-1}^{*}=X^{r-1-l}}{\longrightarrow} \mathcal{F}_{l, k}^{*} \stackrel{X_{0}^{*}=X^{l}}{\longleftarrow} \mathcal{F}_{-l, k}^{*} \stackrel{X_{1}^{*}=X^{r-1-l}}{\longleftarrow} \cdots \\
\ldots \stackrel{Y_{-2}=Y^{k}}{\longrightarrow} \mathcal{F}_{l, 2 r-k} \stackrel{Y_{-1}=Y^{r-k}}{\longrightarrow} \mathcal{F}_{l, k} \stackrel{Y_{0}=Y^{k}}{\longrightarrow} \mathcal{F}_{l,-k} \stackrel{Y_{1}=Y^{r-k}}{\longrightarrow} \cdots \\
\ldots \stackrel{Y_{-2}^{*}=Y^{k}}{\longleftarrow} \mathcal{F}_{l, 2 r-k}^{*} \stackrel{Y_{-1}^{*}=Y^{r-k}}{\longleftarrow} \mathcal{F}_{l, k}^{*} \stackrel{Y_{0}^{*}=Y^{k}}{\longleftarrow} \mathcal{F}_{l,-k}^{*} \stackrel{Y_{1}^{*}=Y^{r-k}}{\longleftarrow} \cdots
\end{gathered}
$$

We assume the following cohomological properties.

$$
\begin{aligned}
\operatorname{Ker} X_{j} / \operatorname{Im} X_{j-1} & =0 \text { if } j \neq 0, \\
\operatorname{Ker} X_{j-1}^{*} / \operatorname{Im} X_{j}^{*} & =0 \text { if } j \neq 0, \\
\operatorname{Ker} X_{0} & =\operatorname{Ker} Y_{0}, \\
\operatorname{Im} X_{-1} & =\operatorname{Im} Y_{-1}, \\
\operatorname{Ker} Y_{j} / \operatorname{Im} Y_{j-1} & =0 \text { if } j \neq 0, \\
\operatorname{Ker} Y_{j-1}^{*} / \operatorname{Im} Y_{j}^{*} & =0 \text { if } j \neq 0, \\
\operatorname{Ker} X_{-1}^{*} & =\operatorname{Ker} Y_{-1}^{*}, \\
\operatorname{Im} X_{0}^{*} & =\operatorname{Im} Y_{0}^{*} .
\end{aligned}
$$

We will make the identification,

$$
\begin{aligned}
\mathcal{L}_{l, k} & =\operatorname{Ker} X_{0} / \operatorname{Im} X_{-1}, \\
\mathcal{L}_{l, k}^{*} & =\operatorname{Ker} X_{-1}^{*} / \operatorname{Im} X_{0}^{*},
\end{aligned}
$$

and conjecture that the coupling

$$
\mathcal{L}_{l, k}^{*} \times \mathcal{L}_{l, k} \rightarrow \mathbf{C}
$$

induced from the coupling

$$
\mathcal{F}_{l, k}^{*} \times \mathcal{F}_{l, k} \rightarrow \mathbf{C}
$$

is non-degenerate.

In summary, we have bosonized the half-infinite transfer matrices $\Phi^{(k \pm 1, k)}(\zeta)$ and $\Phi^{*(k, k \pm 1)}\left(\zeta^{-1}\right)$ by means of the type I vertex operators $\Phi_{ \pm}(\zeta)$ and $\Phi_{ \pm}^{*}\left(\zeta^{-1}\right)$, and also introduced type II vertex operators, which will play the role of creation operators of particles as we will see in the following section. 


\section{The Boundary States}

\subsection{Construction in the Fock space}

In Section 2, we chose the normalisation of the bulk and boundary weights such that the maximal eigenvalues of the bulk and boundary transfer matrices in the large lattice limit were 1 . In this section we describe how to construct the corresponding eigenvector in the bosonic Fock space $\mathcal{F}_{k, k}$ or $\mathcal{F}_{k-1, k}$, depending on whether $b>0$ or $b<0$. We will then show that the eigenvector so constructed actually determines a non-zero residue class in the corresponding subquotient $\mathcal{L}_{k, k}$ or $\mathcal{L}_{k-1, k}$.

The maximal eigenvectors $|k, k\rangle_{B}^{c}$ and $|k-1, k\rangle_{B}^{c}$ are defined in both regions A and B (defined in Section 2.3) by,

$$
\begin{aligned}
T_{B}^{(k)}(\zeta)|k, k\rangle_{B}^{c} & =|k, k\rangle_{B}^{c} \in \mathcal{F}_{k, k}, \quad \text { for } b>0, \\
T_{B}^{(k)}(\zeta)|k-1, k\rangle_{B}^{c} & =|k-1, k\rangle_{B}^{c} \in \mathcal{F}_{k-1, k}, \quad \text { for } b<0 .
\end{aligned}
$$

We make the Ansatz that

$$
|k-i, k\rangle_{B}^{c}=e^{F_{i}^{c, k}}|k-i, k\rangle, \text { for } i=0,1
$$

where

$$
F_{i}^{c, k}=-\frac{1}{2} \sum_{m>0} \frac{[2 m]_{x}[r m]_{x}}{m[m]_{x}[(r-1) m]_{x}} \beta_{-m}^{2}+\sum_{m>0} \frac{D_{m, i}^{c, k}}{m} \beta_{-m} .
$$

The adjoint action of $e^{F_{i}^{c, k}}$ on the bosonic oscillator modes is that of a Bogoliubov transformation. Namely,

$$
\begin{aligned}
e^{-F_{i}^{c, k}} \beta_{m} e^{F_{i}^{c, k}} & =\beta_{m}-\beta_{-m}+\frac{[m]_{x}[(r-1) m]_{x}}{[2 m]_{x}[r m]_{x}} D_{m, i}^{c, k}, \\
e^{-F_{i}^{c, k}} \beta_{-m} e^{F_{i}^{c, k}} & =\beta_{-m},
\end{aligned}
$$

where $m>0$. The coefficients $D_{m, i}^{c, k}$ are determined by solving conditions (4.1) and (4.2). Rewriting these conditions in terms of the bosonic vertex operator expression for $T_{B}^{(k)}(\zeta)$ (2.12), and using the inversion property (3.51), we obtain

$$
\begin{aligned}
& K_{>}^{(c)}\left(k+\varepsilon_{k}^{k} \mid u\right) \Phi_{\varepsilon}(\zeta) e^{F_{0}^{c, k}}|k, k\rangle=\Phi_{\varepsilon}\left(\zeta^{-1}\right) e^{F_{0}^{c, k}}|k, k\rangle \quad \text { for } b>0, \\
& K_{<}^{(c)}\left(k+\varepsilon_{k}^{k} \mid u\right) \Phi_{\varepsilon}(\zeta) e^{F_{1}^{c, k}}|k-1, k\rangle=\Phi_{\varepsilon}\left(\zeta^{-1}\right) e^{F_{1}^{c, k}}|k-1, k\rangle \quad \text { for } b<0 .
\end{aligned}
$$

Solving these conditions for $\varepsilon=+$, we find

$$
\begin{aligned}
D_{m, 0}^{c, k} & =-\frac{[(k-1) m]_{x}[(r-2 c-k) m]_{x}^{+}}{[(r-1) m]_{x}}-\theta_{m}\left(\frac{[m / 2]_{x}[r m / 2]_{x}^{+}}{[(r-1) m / 2]_{x}}\right), \\
D_{m, 1}^{c, k} & =\frac{[r+1-k) m]_{x}[(2 c+k) m]_{x}^{+}}{[(r-1) m]_{x}}-\theta_{m}\left(\frac{[m / 2]_{x}[r m / 2]_{x}^{+}}{[(r-1) m / 2]_{x}}\right) \\
& =D_{m, 0}^{c, k}+\frac{[r m]_{x}[(2 c+1) m]_{x}^{+}}{[(r-1) m]_{x}}
\end{aligned}
$$


where

$$
\begin{aligned}
& {[k]_{x}^{+}=x^{k}+x^{-k},} \\
& \theta_{m}(x)= \begin{cases}x & \text { if } m \text { is even; } \\
0 & \text { otherwise. }\end{cases}
\end{aligned}
$$

In order to show that (4.6) and (4.7) hold for $\varepsilon=-$, with $D_{m, i}^{c, k}$ given by $(4.8)$ and (4.9), we first consider (4.6) for $k=1$. We find that,

$$
\begin{aligned}
& e^{-F_{0}^{c, 1}} \Phi_{-}(\zeta) e^{F_{0}^{c, 1}}|1,1\rangle=\lambda^{-1} \zeta^{\frac{3 r-1}{4 r}} f(\zeta)^{-1} \\
& \times \oint \frac{d z}{2 \pi i z}\left(z-z^{-1}\right) \frac{\left(x^{2 r-1} \zeta z ; x^{2 r}\right)_{\infty}\left(x^{2 r-1} \zeta / z ; x^{2 r}\right)_{\infty}}{\left(x \zeta z ; x^{2 r}\right)_{\infty}\left(x \zeta / z ; x^{2 r}\right)_{\infty}} e^{\sum_{m<0} \frac{\beta_{m}}{m}\left(\left(\zeta^{m}+\zeta^{-m}\right)-\left(\xi^{m}+\xi^{-m}\right)\left(z^{m}+z^{-m}\right)\right)}|1,1\rangle=0 .
\end{aligned}
$$

The integral vanishes because of the anti-symmetry of the integrand under the change of variables $z \rightarrow z^{-1}$. The proof that (4.6) and (4.7) are valid for $\varepsilon=-$ and general $k$ is inductive and is given in Appendix B.

In a similar manner we construct dual maximal eigenstates in the Fock spaces $\mathcal{F}_{k, k}^{*}$ and $\mathcal{F}_{k-1, k}^{*}$. These states are determined by the requirements,

$$
\begin{aligned}
{ }_{B}^{c}\langle k, k| T_{B}^{(k)}(\zeta) & ={ }_{B}^{c}\langle k, k| \in \mathcal{F}_{k, k}^{*} \quad \text { for } b>0, \\
{ }_{B}^{c}\langle k-1, k| T_{B}^{(k)}(\zeta) & ={ }_{B}^{c}\langle k-1, k| \in \mathcal{F}_{k-1, k}^{*} \quad \text { for } b<0 .
\end{aligned}
$$

As above we make the Ansatz that

$$
{ }_{B}^{c}\langle k-i, k|=\langle k-i, k| e^{G_{i}^{c, k}},
$$

where

$$
G_{i}^{c, k}=-\frac{1}{2} \sum_{m>0} x^{4 m} \frac{[2 m]_{x}[r m]_{x}}{m[m]_{x}[(r-1) m]_{x}} \beta_{m}^{2}+\sum_{m>0} \frac{E_{m, i}^{c, k}}{m} \beta_{m} .
$$

$e^{G_{i}^{c, k}}$ now produces the Bogoliubov transformation

$$
\begin{aligned}
e^{G_{i}^{c, k}} \beta_{-m} e^{-G_{i}^{c, k}} & =\beta_{-m}-x^{4 m} \beta_{m}+\frac{[m][(r-1) m]}{[2 m][r m]} E_{m, i}^{c, k}, \\
e^{G_{i}^{c, k}} \beta_{m} e^{-G_{i}^{c, k}} & =\beta_{m},
\end{aligned}
$$

where $m>0$. In terms of bosonized vertex operators, (4.13) and (4.14) become

$$
\begin{array}{r}
{ }_{B}^{c}\langle k, k| \Phi_{\varepsilon}^{*}\left(\zeta^{-1}\right) K_{>}^{(c)}\left(k+\varepsilon_{k}^{k} \mid u\right)={ }_{B}^{c}\langle k, k| \Phi_{\varepsilon}^{*}(\zeta) \text { for } b>0, \\
{ }_{B}^{c}\langle k-1, k| \Phi_{\varepsilon}^{*}\left(\zeta^{-1}\right) K_{<}^{(c)}\left(k+\varepsilon_{k}^{k} \mid u\right)={ }_{B}^{c}\langle k-1, k| \Phi_{\varepsilon}^{*}(\zeta) \text { for } b<0 .
\end{array}
$$

Solving these equation, we find

$$
\begin{aligned}
E_{m, 0}^{c, k} & =-x^{2 m} \frac{[(k+1) m][(r-2 c-k) m]_{x}^{+}}{[(r-1) m]}+x^{2 m} \theta_{m}\left(\frac{[m / 2]_{x}[r m / 2]_{x}^{+}}{[(r-1) m / 2]_{x}}\right) \\
E_{m, 1}^{c, k} & =x^{2 m} \frac{[(r-k-1) m][(2 c+k) m]_{x}^{+}}{[(r-1) m]}+x^{2 m} \theta_{m}\left(\frac{[m / 2]_{x}[r m / 2]_{x}^{+}}{[(r-1) m / 2]_{x}}\right) \\
& =E_{m, 0}^{c, k}+x^{2 m} \frac{[r m]_{x}[(1-2 c) m]_{x}^{+}}{[(r-1) m]}
\end{aligned}
$$




\subsection{Boundary vacuum states in the subquotients}

We have constructed maximal eigenvectors $|k-i, k, k\rangle_{B}^{c} \in \mathcal{F}_{k-i, k}$, and dual eigenvectors ${ }_{B}^{c}\langle k-i, k, k| \in$ $\mathcal{F}_{k-i, k}^{*}$. In order to establish that these vectors actually give rise to the eigenvectors in the subquotients $\mathcal{L}_{k-i, k}=\operatorname{Ker} X_{0} / \operatorname{Im} X_{-1}$ and $\mathcal{L}_{k-i, k}^{*}=\operatorname{Ker} X_{-1}^{*} / \operatorname{Im} X_{0}^{*}$ respectively, it is sufficient to show that,

$$
\begin{aligned}
|k-i, k\rangle_{B}^{c} & \in \operatorname{Ker} X_{0}=\operatorname{Ker} Y_{0} \subset \mathcal{F}_{k-i, k}, \\
{ }_{B}^{c}\langle k-i, k| & \in \operatorname{Ker} X_{-1}^{*}=\operatorname{Ker} Y_{-1}^{*} \subset \mathcal{F}_{k-i, k}^{*}, \\
{ }_{B}^{c}\langle k-i, k \mid k-i, k\rangle_{B}^{c} & \neq 0 .
\end{aligned}
$$

If these three conditions are true then it is simple to show that

$$
{ }_{B}^{c}\langle k-i, k|P| k-i, k\rangle_{B}^{c}={ }_{B}^{c}\langle\overline{k-i, k}|P| \overline{k-i, k}\rangle_{B}^{c},
$$

where $|\overline{k-i, k}\rangle_{B}^{c}$ is the residue class of $|k-i, k\rangle_{B}^{c}$ in $\mathcal{L}_{k-i, k}, \quad{ }_{B}^{c}\langle\overline{k-i, k}|$ is the residue class of ${ }_{B}^{c}\langle k-i, k|$ in $\mathcal{L}_{k-i, k}^{*}$, and $P$ is an operator that commutes with the coboundary operators $X$ and $Y$. Local operators of the of the theory are expressed in terms of products of vertex operators $\Phi_{\varepsilon}(z)$, and thus an example of an operator $P$. Hence if (4.24) - 4.25) hold we can calculate local correlation functions of the theory as matrix elements of the form ${ }_{B}^{c}\langle k-i, k|P| k-i, k\rangle_{B}^{c}$. Form factors are also in this class because they are the matrix elements of products of type I and type II vertex operators which commute with $X$ and $Y$ respectively - see (3.61).

We establish condition (4.24) as follows. For $|1,1\rangle_{B}^{c}=e^{F_{0}^{c, 1}}|1,1\rangle$, one can show the result directly. Namely,

$$
e^{-F_{0}^{c, 1}} X e^{F_{0}^{c, 1}}|1,1\rangle \propto \oint \frac{d z}{2 \pi i z}\left(z-z^{-1}\right) e^{\sum_{m>0} \frac{\alpha_{-m}}{m} \frac{[m]}{[2 m]}\left(z^{m}+z^{-m}\right)}|-1,1\rangle=0 .
$$

This integral is zero because of the anti-symmetry of the integrand under $z \rightarrow z^{-1}$. For general $|k-i, k\rangle_{B}^{c}$ this technique is rather complicated and it is simpler to proceed by other means. We observe that different boundary states are related to each other by the action of vertex operators. In particular we find that

$$
\begin{aligned}
\Phi_{+}\left(x^{2 c}\right)|k, k\rangle_{B}^{c} & \sim|k, k+1\rangle_{B}^{c-1}, \\
\operatorname{Res}_{\xi=-x^{1+2 c}} \Psi_{+}^{*}(\xi)|k-1, k\rangle_{B}^{c} & \sim|k, k\rangle_{B}^{c} .
\end{aligned}
$$

Here $\sim$ means equal up to scalar functions. We shall discuss (4.30) and other relations in more detail in Section 6. These relations are useful in this context because the screening charge $X$ commutes with $\Phi(\xi)$, and $Y$ commutes with $\Psi^{*}(\xi)$. Thus

$$
\begin{aligned}
X|1,2\rangle_{B}^{c} & \sim \Phi_{+}\left(x^{2(c+1)}\right) X|1,1\rangle_{B}^{c+1}=0 \\
Y^{2}|2,2\rangle_{B}^{c} & \sim \operatorname{Res}_{\xi=-x^{1+2 c}} \Psi_{+}^{*}(\xi) Y^{2}|1,2\rangle_{B}^{c}=0
\end{aligned}
$$

where $|1,1\rangle_{B}^{c+1}$ is an analytic continuation in $c$ of $|1,1\rangle_{B}^{c}$. By repeatedly applying vertex operators to construct the general state $|k-i, k\rangle_{B}^{c}$, we can show that $|k-i, k\rangle_{B}^{c} \in \operatorname{Ker} X_{0}=\operatorname{Ker} Y_{0}$ as required. 
We establish condition (4.25) in a completely analogous manner. First we show the result for ${ }_{B}^{c}\langle r-2, r-1|$ directly using,

$\langle r-2, r-1| e^{G_{1}^{c, r-1}} X e^{-G_{1}^{c, r-1}} \propto\langle r, r-1| \oint \frac{d z}{2 \pi i z}\left(z x^{2}-z^{-1} x^{-2}\right) e^{-\sum_{m>0} \frac{x^{2 m} \alpha_{m}}{m} \frac{[2 m]}{[m]}\left(z^{m} x^{2 m}+z^{-m} x^{-2 m}\right)}=0$.

This integral vanishes because of anti-symmetry under the changes of variables $z x^{2} \rightarrow z^{-1} x^{-2}$. Again, different dual states are related to each other under the action of vertex operators. We find,

$$
\begin{aligned}
{ }_{B}^{c}\langle k-1, k| \Phi_{-}^{*}\left(x^{2 c}\right) & \sim{ }_{B}^{c+1}\langle k-1, k-1|, \\
\operatorname{Res}_{\xi=-x^{1-2 c}}{ }_{B}^{c}\langle k, k| \Psi_{-}\left(\xi^{-1}\right) & \sim{ }_{B}^{c}\langle k-1, k| .
\end{aligned}
$$

Thus

$$
\begin{aligned}
{ }_{B}^{c}\langle r-2, r-2| X & \sim{ }_{B}^{c-1}\langle r-2, r-1| X \Phi_{-}^{*}\left(x^{2(c-1)}\right)=0 \\
{ }_{B}^{c}\langle r-3, r-2| Y^{2} & \sim \operatorname{Res}_{\xi=-x^{1-2 c}}{ }_{B}^{c}\langle r-2, r-2| Y^{2} \Psi_{-}\left(\xi^{-1}\right)=0 \quad \text { etc. }
\end{aligned}
$$

The general result ${ }_{B}^{c}\langle k-i, k| \in \operatorname{Ker} X_{-1}^{*}=\operatorname{Ker} Y_{-1}^{*}$ follows.

Finally, we show (4.26) by computing ${ }_{B}^{c}\langle k-i, k \mid k-i, k\rangle_{B}^{c}$ explicitly. We do this by using a decomposition of the identity on $\mathcal{F}_{l, k}$ which employs coherent states. Define the coherent states

$$
\begin{aligned}
|\xi\rangle & =e^{\sum_{m>0} \xi_{m} \kappa_{m} \beta_{-m}}|l, k\rangle, \quad\langle\bar{\xi}|=\langle l, k| e^{\sum_{m>0} \bar{\xi}_{m} \kappa_{m} \beta_{m}}, \quad \text { where } \\
\kappa_{m} & =\frac{1}{m} \frac{[2 m]_{x}[r m]_{x}}{[m]_{x}[(r-1) m]_{x}}
\end{aligned}
$$

It is simple to show that

$$
\begin{aligned}
\beta_{m}|\xi\rangle & =\xi_{m}|\xi\rangle, \quad\langle\bar{\xi}| \beta_{-m}=\langle\bar{\xi}| \bar{\xi}_{m} \\
\langle\bar{\xi} \mid \xi\rangle & =e^{\sum_{m>0} \kappa_{m} \bar{\xi}_{m} \xi_{m}}
\end{aligned}
$$

Its is possible to decompose the identity on $\mathcal{F}_{l, k}$ as

$$
\operatorname{id}_{\mathcal{F}_{l, k}}=\int_{-\infty}^{\infty} \prod_{m>0} d \xi_{m} d \bar{\xi}_{m} \kappa_{m} e^{-\sum_{m>0} \kappa_{m} \bar{\xi}_{m} \xi_{m}}|\xi\rangle_{l, k} l, k\langle\bar{\xi}| .
$$

This is shown using the Gaussian integral

$$
\begin{aligned}
& \int_{-\infty}^{\infty} \prod_{m>0} d \xi_{m} d \bar{\xi}_{m} \kappa_{m} e^{\left(-\frac{1}{2} \sum_{m>0} \kappa_{m}\left(\bar{\xi}_{m}, \xi_{m}\right) \mathcal{A}_{m}\left(\begin{array}{l}
\bar{\xi}_{m} \\
\xi_{m}
\end{array}\right)+\sum_{m>0}\left(\bar{\xi}_{m}, \xi_{m}\right) \mathcal{B}_{m}\right)} \\
& =\prod_{m>0}\left(-\operatorname{det}\left(\mathcal{A}_{m}\right)\right)^{-\frac{1}{2}} e^{\left(\frac{1}{2} \sum_{m>0} \kappa_{m}^{-1} \mathcal{B}_{m}^{t} \mathcal{A}_{m}^{-1} \mathcal{B}_{m}\right)} .
\end{aligned}
$$

Inserting this decomposition of the identity between $e^{G_{i}^{c, k}} e^{F_{i}^{c, k}}$ and using (4.38), we find,

$$
\begin{aligned}
& { }_{B}^{c}\langle k-i, k \mid k-i, k\rangle_{B}^{c}=\left\langle k-i, k\left|e^{G_{i}^{c, k}} e^{F_{i}^{c, k}}\right| k-i, k\right\rangle \\
& =\frac{1}{\left(x^{4} ; x^{4}\right)_{\infty}^{\frac{1}{2}}} \exp \left(-\frac{1}{2} \sum_{m>0} \frac{x^{4 m}\left(D_{m, i}^{c, k}\right)^{2}-2 D_{m, i}^{c, k} E_{m, i}^{c, k}+\left(E_{m, i}^{c, k}\right)^{2}}{\kappa_{m}\left(1-x^{4 m}\right) m^{2}}\right),
\end{aligned}
$$


For $i=0$ this is equal to

${ }_{B}^{c}\langle k, k \mid k, k\rangle_{B}^{c}=\frac{1}{\left(x^{6} ; x^{8}\right)_{\infty}} \frac{\left(x^{8} ; x^{8}, x^{2 r}\right)_{\infty}\left(x^{4} ; x^{8}, x^{2(r-1)}\right)_{\infty}}{\left(x^{8} ; x^{8}, x^{2(r-1)}\right)_{\infty}\left(x^{4} ; x^{8}, x^{2 r}\right)_{\infty}}$

$\times \frac{\left(x^{4 r-4 c-2 k} ; x^{4}, x^{2(r-1)}\right)_{\infty}\left(x^{4 c+2 k} ; x^{4}, x^{2(r-1)}\right)_{\infty}\left(x^{2 r} ; x^{4}, x^{2(r-1)}\right)_{\infty}^{2}\left(x^{2 r+4-2 k} ; x^{4}, x^{2 r}\right)_{\infty}\left(x^{2 r+4+2 k} ; x^{4}, x^{2 r}\right)_{\infty}}{\left(x^{2+4 r-4 c-2 k} ; x^{4}, x^{2 r}\right)_{\infty}\left(x^{2+4 c+2 k} ; x^{4}, x^{2 r}\right)_{\infty}\left(x^{2+2 r} ; x^{4}, x^{2 r}\right)_{\infty}^{2}\left(x^{2 r+2-2 k} ; x^{4}, x^{2(r-1)}\right)_{\infty}\left(x^{2 r+2+2 k} ; x^{4}, x^{2(r-1)}\right)_{\infty}}$

$\times \frac{\left(x^{4 r+4-4 k-4 c} ; x^{8} ; x^{2 r}\right)_{\infty}\left(x^{4+4 c} ; x^{8} ; x^{2 r}\right)_{\infty}\left(x^{4 r+4-4 c} ; x^{8} ; x^{2 r}\right)_{\infty}\left(x^{4+4 k+4 c} ; x^{8} ; x^{2 r}\right)_{\infty}}{\left(x^{4 r+2-4 k-4 c} ; x^{8} ; x^{2(r-1)}\right)_{\infty}\left(x^{2+4 c} ; x^{8} ; x^{2(r-1)}\right)_{\infty}\left(x^{4 r+2-4 c} ; x^{8} ; x^{2(r-1)}\right)_{\infty}\left(x^{2+4 k+4 c} ; x^{8} ; x^{2(r-1)}\right)_{\infty}}$.

From the explicit form of $D_{m, i}^{c, k}$ and $E_{m, i}^{c, k}$, we see we can recover the $i=1$ normalisation from the $i=0$ result by the transformation $(c, k) \rightarrow(c+r, k-r)$.

\section{General Eigenstates and The Boundary S-Matrix}

\subsection{General Eigenstates of $T_{B}^{(k)}(\zeta)$}

We construct general eigenstates of $T_{B}^{(k)}(\zeta)$ by using type II vertex operators. The technique closely parallels that used for the XXZ model [4, 8]. From (2.12) and (3.54) it follows that

$$
\begin{aligned}
T_{B}^{(k)}(\zeta) \Psi_{\varepsilon}^{*}(\xi) & =t(\zeta, \xi) \Psi_{\varepsilon}^{*}(\xi) T_{B}^{(k)}(\zeta), \quad \text { where, } \\
t(\zeta, \xi) & =\chi(\xi / \zeta) \chi\left(\xi \zeta / x^{2}\right)=\chi(\xi / \zeta) \chi(1 /(\xi \zeta)) .
\end{aligned}
$$

From the definition (2.14) of the Hamiltonian $H_{B}^{(k)}$ and the property $T_{B}^{(k)}(1)=$ id (which follows from the definition (2.12)), it also follows that,

$$
\begin{aligned}
{\left[H_{B}^{(k)}, \Psi_{\varepsilon}^{*}(\xi)\right] } & =\epsilon(\xi) \Psi_{\varepsilon}^{*}(\xi), \quad \text { where } \\
\epsilon(\xi) & =\frac{2 I}{\pi} \sinh \left(\frac{\pi I^{\prime}}{I}\right) \operatorname{dn}\left(\frac{2 I \theta}{\pi}\right), \quad \xi=-e^{2 i \theta}
\end{aligned}
$$

Here, $\mathrm{dn}$ is the Jacobi elliptic function with nome $x$, and $I$ and $I^{\prime}$ are the associated half-period magnitudes [2].

Thus it is possible to construct general continuous eigenstates of the form

$$
\begin{aligned}
\Psi_{\varepsilon_{1}}^{*}\left(\xi_{1}\right) \cdots \Psi_{\varepsilon_{n}}^{*}\left(\xi_{n}\right)|k, k\rangle_{B}^{c} & \in \mathcal{F}_{k+\varepsilon_{1}+\cdots+\varepsilon_{n}, k} & \text { for } b>0 \\
\Psi_{\varepsilon_{1}}^{*}\left(\xi_{1}\right) \cdots \Psi_{\varepsilon_{n}}^{*}\left(\xi_{n}\right)|k-1, k\rangle_{B}^{c} & \in \mathcal{F}_{k-1+\varepsilon_{1}+\cdots+\varepsilon_{n}, k} & \text { for } b<0,
\end{aligned}
$$

with $\left|\xi_{i}\right|=1$. The physical picture of these excitations is of $n$ kinks or domain walls that are free, i.e., not bound to the right boundary.

\subsection{The Boundary S-Matrix}

The boundary S-matrix describes the scattering of these kink states off the right boundary. Following [10], we define the boundary S-matrix $M_{\varepsilon}^{(k-i, k)}(\xi ; r, c)$ by

$$
\begin{aligned}
\Psi_{\varepsilon}^{*}(\xi)|k-i, k\rangle_{B}^{c} & =M_{\varepsilon}^{(k-i, k)}(\alpha ; r, c) \Psi_{\varepsilon}^{*}\left(\xi^{-1}\right)|k-i, k\rangle_{B}^{c} \quad \text { for } i=0,1, \\
{ }_{B}^{c}\langle k-i, k| \Psi_{\varepsilon}\left(\xi^{-1}\right) & ={ }_{B}^{c}\langle k-i, k| \Psi_{\varepsilon}(\xi) M_{\varepsilon}^{(k-i, k)}(\alpha ; r, c),
\end{aligned}
$$


where $\xi=x^{2 \alpha}$. Solving condition (5.4) and (5.5) We find,

$$
\begin{aligned}
M_{ \pm}^{(k, k)}(\alpha ; r, c) & =-\xi \frac{\theta_{x^{8}}\left(\xi^{-2} x^{2}\right)}{\theta_{x^{8}}\left(\xi^{2} x^{2}\right)} K_{>}^{\left(c-\frac{1}{2}+\delta\right)}\left(k \pm 1_{k}^{k} \mid \alpha\right), \\
M_{ \pm}^{(k-1, k)}(\alpha ; r, c) & =-\xi \frac{\theta_{x^{8}}\left(\xi^{-2} x^{2}\right)}{\theta_{x^{8}}\left(\xi^{2} x^{2}\right)} K_{<}^{\left(c+\frac{1}{2}+\delta\right)}\left(k \pm 1_{k}^{k} \mid \alpha\right),
\end{aligned}
$$

where $\delta=\frac{\pi i}{2 \ln x}$, and $K^{\prime}$ indicates that $r$ is replaced by $r-1$ in the boundary weights. The boundary S-matrix elements are thus given in terms of the boundary Boltzmann weights (2.18)(2.21) analytically continued to shifted arguments. Again, this is very similar to the situation for the boundary XXZ model [8]. The sole effect of the $\delta$ is to produce the minus sign in the shift $x^{2 c} \rightarrow-x^{2 c \mp 1}$.

In order to discover discrete eigenstates of $T_{B}^{(k)}(\xi)$ other than the maximal ones, we should examine the boundary S-matrix elements in search of simple poles in the physical strip. We identify the boundary physical strip as $x<|\xi|<1$, and find just two simple boundary S-matrix poles which can lie in this region for the allowed values of $c$. Firstly, we find that there is a pole in $M_{-}^{(k, k)}(\alpha ; r, c)$ at $\xi=-x^{1-2 c}$. By explicit calculation, we have shown that this arises from a simple pole $\sim 1 /\left(\xi+x^{1-2 c}\right)$ in $\Psi_{-}^{*}(\xi)|k, k\rangle_{B}^{c}$ with residue

$$
\operatorname{Res}_{\xi=-x^{1-2 c}} \Psi_{-}^{*}(\xi)|k, k\rangle_{B}^{c} \sim|k-1, k\rangle_{B}^{c},
$$

where $|k-1, k\rangle_{B}^{c}$ is an analytic continuation of (4.3) in $c$. Equivalently, this boundary S-matrix pole arises from a pole in ${ }_{B}^{c}\langle k, k| \Psi_{-}\left(\xi^{-1}\right)$ at $\xi=-x^{1-2 c}$. The residue at this pole is

$$
\operatorname{Res}_{\xi=-x^{1-2 c}} \stackrel{c}{B}\langle k, k| \Psi_{-}\left(\xi^{-1}\right) \sim{ }_{B}^{c}\langle k-1, k| .
$$

The second pole that can lie in the boundary physical strip occurs in $M_{+}^{(k-1, k)}(\alpha ; r, c)$ at $\xi=$ $-x^{1+2 c}$. This pole arise from one in $\Psi_{+}^{*}(\xi)|k-1, k\rangle_{B}^{c}$ with the following residue,

$$
\operatorname{Res}_{\xi=-x^{1+2 c}} \Psi_{+}^{*}(\xi)|k-1, k\rangle_{B}^{c} \sim|k, k\rangle_{B}^{c},
$$

or equivalently from

$$
\operatorname{Res}_{\xi=-x^{1+2 c}}{ }_{B}^{c}\langle k-1, k| \Psi_{+}\left(\xi^{-1}\right) \sim{ }_{B}^{c}\langle k, k| .
$$

There are no poles in $M_{+}^{(k, k)}(\alpha ; r, c)$ or $M_{-}^{(k-1, k)}(\alpha ; r, c)$ in the physical strip for either of the parameter regions $A$ or $B$.

We shall now discuss the regions $A$ and $B$ separately and in more detail. First consider regime $A$, in which $x^{2 c}=-x^{2 b},-1<b<1$. For $b>0$ there is a unique ground state with energy eigenvalue zero. This is $|k, k\rangle_{B}^{c}$. For $0<b<\frac{1}{2}$ the pole 5.8$)$ in $M_{-}^{(k, k)}(\alpha ; r, c)$ at $\xi=x^{1-2 b}$ lies in the physical strip. The state $|k-1, k\rangle_{B}^{c}$ can be interpreted as a bound state of a '- kink' with the boundary. The energy of this bound state increases from zero at $b=0$ up to a maximum value of $\epsilon(1)$ at $b=\frac{1}{2}$. This maximum value is equal to the minimum of the energy band of a single free kink state (i.e. one of the form $\Psi_{-}^{*}(\xi)|k, k\rangle_{B}^{c}$ with $|\xi|=1$ ). For $b \geq \frac{1}{2}$ the pole moves off the physical strip, and the state $|k-1, k\rangle_{B}^{c}$ can decay by emitting a kink. For $b<0$ the unique ground state with energy zero is $|k-1, k\rangle_{B}^{c}$. The pole in $M_{+}^{(k-1, k)}(\alpha ; r, c)$ at $\xi=x^{1+2 b}$ lies in the physical 
strip for $-\frac{1}{2}<b<0$. In this region $|k, k\rangle_{B}^{c}$ is a bound state of $|k-1, k\rangle_{B}^{c}$ with a + kink. Again, for $b \leq-\frac{1}{2}$ the pole moves off the physical strip and $|k, k\rangle_{B}^{c}$ is unstable against emission of a free kink.

Now consider regime $B$, which is parameterised by $x^{2 c}=x^{2 b},-1<b<1$. For $0<b<\frac{1}{2}$ the pole 5.8$)$ in $M_{-}^{(k, k)}(\alpha ; r, c)$ at $\xi=-x^{1-2 b}$ still lies in the physical strip, and the energy of the bound state $|k-1, k\rangle_{B}^{c}$ is outside the energy band of a free kink. However, in this case, the bound state energy is greater than the maximum energy $\epsilon(-1)$ of a free kink. As $b$ increases the energy of the bound state $|k, k\rangle_{B}^{c}$ decreases and reaches $\epsilon(-1)$ when $b$ is equal to the critical value $\frac{1}{2}$. For $b \geq \frac{1}{2}$ the pole moves off the physical strip and $|k-1, k\rangle_{B}^{c}$ can decay by emission of a kink. This mechanism for the stability of a bound state in a lattice model was also observed for the XXZ model in [8]. For $b<0$, again, and for similar reasons, there is a stable bound state $|k, k\rangle_{B}^{c}$ when $-\frac{1}{2}<b<0$.

We remark that the normalisation (4.39) also confirms the above discussion, because ${ }_{B}^{c}\langle k, k \mid k, k\rangle_{B}^{v}$ has has a pole at $x^{2 c}= \pm x^{-1}$, and ${ }_{B}^{c}\langle k-1, k \mid k-1, k\rangle_{B}^{v}$ has a pole at $x^{2 c}= \pm x$.

\section{$6 \quad$ The Scaling Limit}

In this section we consider the $x \rightarrow 1$ scaling limit of the bulk and boundary S-matrices. Parameterising $\xi=x^{2 \alpha}$, we shall identify $\pi i \alpha$ with the usual rapidity parameter $\theta$ in this limit. Our boundary physical strip becomes $0<\operatorname{Im} \theta<\pi / 2$ as in $\mid 14$ ]. The pole at $\xi=x^{1-2|b|}$ in region $\mathrm{A}$ thus lies on the imaginary axis in the $\theta$ plane. It is a physical strip pole that may be interpreted as a bound state. The pole at $\xi=-x^{1-2|b|}$ in region B moves off to infinity in the scaling limit. The energy of the associated state becomes infinite, as the one particle energy band becomes infinity high, and it should no longer be considered as a bound state. It is for region A that we shall construct the scaling limit of the bulk and boundary S-matrices.

\subsection{A gauge transformation}

Before taking the scaling limit we carry out the following gauge transformation:

$$
\begin{aligned}
\tilde{S}\left(\begin{array}{cc}
m_{4} & m_{1} \\
m_{3} & m_{2}
\end{array} \mid \alpha\right) & =-\left(\frac{\left[m_{4}\right]^{\prime}\left[m_{2}\right]^{\prime}}{\left[m_{1}\right]^{\prime}\left[m_{3}\right]^{\prime}}\right)^{\alpha / 2} \sqrt{\frac{\left[m_{1}\right]^{\prime}}{\left[m_{3}\right]^{\prime}}} W^{\prime}\left(\begin{array}{cc}
m_{4} & m_{1} \\
m_{3} & m_{2}
\end{array} \mid \alpha\right), \\
\tilde{M}_{\varepsilon}^{(k-i, k)}(\alpha) & =\left(\frac{[k-i+\varepsilon]^{\prime}}{[k-i]^{\prime}}\right)^{\alpha} M_{\varepsilon}^{(k-i, k)}(\alpha), \\
\tilde{\Psi}^{*(l+\varepsilon, l)}(\xi) & =\left(\frac{[l+\varepsilon]^{\prime}}{[l]^{\prime}}\right)^{\alpha / 2} \sqrt{\frac{[1]^{\prime}}{[l+\varepsilon]^{\prime}}} \Psi^{*(l+\varepsilon, l)}(\xi), \\
\tilde{\Psi}_{\varepsilon}(\xi) & =\tilde{\Psi}_{-\varepsilon}^{*}\left(x^{2} \xi\right),
\end{aligned}
$$

\footnotetext{
${ }^{1}$ In [10], the boundary physical strip was at one point incorrectly identified as $0<\operatorname{Im} \theta<\pi$. This was corrected
} in 14 . 
where $\xi=x^{2 \alpha}$. The effect of this gauge transformation is to yield bulk and boundary S-matrices $\tilde{S}$ and $\tilde{M}$ that have the conventional crossing and boundary crossing/unitarity relations (a rather similar gauge transformation was carried out in reference [15] in order to yield a crossing symmetric S-matrix from RSOS Boltzmann weights). Written in terms of these gauge transformed objects, equations (3.53), (5.4) and (5.5) become,

$$
\begin{aligned}
\tilde{\Psi}_{\varepsilon_{2}}^{*}\left(\xi_{2}\right) \tilde{\Psi}_{\varepsilon_{1}}^{*}\left(\xi_{1}\right) & =\sum_{\varepsilon_{1}^{\prime}+\varepsilon_{2}^{\prime}=\varepsilon_{1}+\varepsilon_{2}} \tilde{S}\left(\begin{array}{cc}
L+\varepsilon_{1}+\varepsilon_{2} & L+\varepsilon_{2}^{\prime} \\
L+\varepsilon_{1} & L
\end{array} \mid \alpha_{2}-\alpha_{1}\right) \tilde{\Psi}_{\varepsilon_{1}^{\prime}}^{*}\left(\xi_{1}\right) \tilde{\Psi}_{\varepsilon_{2}^{\prime}}^{*}\left(\xi_{2}\right), \\
\tilde{\Psi}_{\varepsilon}^{*}(\xi)|k-i, k\rangle_{B}^{c} & =\tilde{M}_{\varepsilon}^{(k-i, k)}(\alpha ; r, c) \tilde{\Psi}_{\varepsilon}^{*}\left(\xi^{-1}\right)|k-i, k\rangle_{B}^{c} \quad \text { for } i=0,1, \\
{ }_{B}^{c}\langle k-i, k| \tilde{\Psi}_{\varepsilon}\left(\xi^{-1}\right) & ={ }_{B}^{c}\langle k-i, k| \tilde{\Psi}_{\varepsilon}(\xi) \tilde{M}_{\varepsilon}^{(k-i, k)}(\alpha ; r, c) .
\end{aligned}
$$

The S-matrix (6.1) obeys (2.4) and (2.5) with the change $W \rightarrow \tilde{S}$. The crossing relation is modified to the more conventional

$$
\tilde{S}\left(\begin{array}{ll}
d & c \\
a & b
\end{array} \mid \alpha\right)=\tilde{S}\left(\begin{array}{ll}
a & d \\
b & c
\end{array} \mid 1-\alpha\right) .
$$

The properties of the boundary S-matrix $\tilde{M}$ can either be read off from the explicit expressions (5.6) and (5.7), or derived using the definition (6.6) and properties (6.5) and (3.57) of vertex operators. They are:

$\sum_{\varepsilon_{1}^{\prime \prime}+\varepsilon_{2}^{\prime \prime}=\varepsilon_{1}+\varepsilon_{2}} \tilde{S}\left(\begin{array}{ll}l+\varepsilon_{1}+\varepsilon_{2} & l+\varepsilon_{1}^{\prime \prime} \\ l+\varepsilon_{2} & l\end{array} \mid \alpha_{1}-\alpha_{2}\right) \tilde{S}\left(\begin{array}{ll}l+\varepsilon_{1}+\varepsilon_{2} & l+\varepsilon_{2}^{\prime} \\ l+\varepsilon_{1}^{\prime \prime} & l\end{array} \mid \alpha_{1}+\alpha_{2}\right) \tilde{M}_{\varepsilon_{1}^{\prime \prime}}^{(l, k)}\left(\xi_{1}\right) \tilde{M}_{\varepsilon_{2}^{\prime}}^{(l, k)}\left(\xi_{2}\right)=$ $\sum_{\varepsilon_{1}^{\prime \prime}+\varepsilon_{2}^{\prime \prime}=\varepsilon_{1}+\varepsilon_{2}} \tilde{S}\left(\begin{array}{ll}l+\varepsilon_{1}+\varepsilon_{2} & l+\varepsilon_{1}^{\prime \prime} \\ l+\varepsilon_{2} & l\end{array} \mid \alpha_{1}+\alpha_{2}\right) \tilde{S}\left(\begin{array}{ll}l+\varepsilon_{1}+\varepsilon_{2} & l+\varepsilon_{2}^{\prime} \\ l+\varepsilon_{1}^{\prime \prime} & l\end{array} \mid \alpha_{1}-\alpha_{2}\right) \tilde{M}_{\varepsilon_{2}}^{(l, k)}\left(\xi_{2}\right) \tilde{M}_{\varepsilon_{1}^{\prime \prime}}^{(l, k)}\left(\xi_{1}\right)$,

where $\varepsilon_{1}, \varepsilon_{2}, \varepsilon_{1}^{\prime}$ and $\varepsilon_{2}^{\prime}$ are fixed with $\varepsilon_{1}+\varepsilon_{2}=\varepsilon_{1}^{\prime}+\varepsilon_{2}^{\prime}$, and $l=k$ or $k-1$,

$$
\begin{aligned}
& \tilde{M}_{\varepsilon}^{(l, k)}(\xi) \tilde{M}_{\varepsilon}^{(l, k)}\left(\xi^{-1}\right)=1, \\
& \tilde{M}_{\varepsilon}^{(l, k)}\left(x^{2} \xi^{-1}\right)=\sum_{\varepsilon^{\prime}} \tilde{S}\left(\begin{array}{ll}
l & l+\varepsilon^{\prime} \\
l+\varepsilon & l
\end{array} \mid \xi^{2} x^{-2}\right) \tilde{M}_{\varepsilon^{\prime}}^{(l, k)}(\xi) .
\end{aligned}
$$

\subsection{Scaling behaviour}

The S-matrices are constructed in terms of functions that have the following behaviour in the limit $p \rightarrow 1:$

$$
\begin{aligned}
\left(p^{z} ; p\right)_{\infty} & \rightarrow(1-p)^{(1-z)} \frac{(p ; p)_{\infty}}{\Gamma(z)} \\
\frac{\Theta_{p}\left(p^{z}\right)}{\Theta_{p}\left(p^{z^{\prime}}\right)} & \rightarrow \frac{\sin (\pi z)}{\sin \left(\pi z^{\prime}\right)}
\end{aligned}
$$

We shall consider the two cases $r=4$ and $r=5$, separately, and in detail. The scaling limit of the $r=4$ model should correspond to the massive $\phi_{(1,3)}$ perturbation of the critical Ising model [10]. 
That of $r=5$ should correspond to that of the massive $\phi_{(1,3)}$ perturbation of the tri-critical Ising model [16, 11].

\subsection{The $r=4$ model}

For $r=4$, there are just two S-matrix elements. Reading off from equations (6.5), (6.1), and (2.1)-(2.3), we find

$$
\begin{aligned}
& \tilde{\Psi}_{+}^{*}\left(\xi_{2}\right) \tilde{\Psi}_{-}^{*}\left(\xi_{1}\right)=\tilde{S}\left(\begin{array}{cc}
2 & 1 \\
1 & 2
\end{array} \mid \alpha_{2}-\alpha_{1}\right) \tilde{\Psi}_{+}^{*}\left(\xi_{1}\right) \tilde{\Psi}_{-}^{*}\left(\xi_{2}\right), \quad \text { on } \mathcal{F}_{2, k} \\
& \tilde{\Psi}_{-}^{*}\left(\xi_{2}\right) \tilde{\Psi}_{+}^{*}\left(\xi_{1}\right)=\tilde{S}\left(\begin{array}{cc}
1 & 2 \\
2 & 1
\end{array} \mid \alpha_{2}-\alpha_{1}\right) \tilde{\Psi}_{-}^{*}\left(\xi_{1}\right) \tilde{\Psi}_{+}^{*}\left(\xi_{2}\right), \quad \text { on } \mathcal{F}_{1, k},
\end{aligned}
$$

where

$$
\tilde{S}\left(\begin{array}{ll}
2 & 1 \\
1 & 2
\end{array} \mid \alpha_{2}-\alpha_{1}\right)=\tilde{S}\left(\begin{array}{ll|l}
1 & 2 \\
2 & 1
\end{array} \mid \alpha_{2}-\alpha_{1}\right)=-1 .
$$

This is the behaviour expected for a free fermion theory. In order to define the scaling limit of the boundary S-matrix elements given by (6.2), (5.6) and (5.7), we must scale $\xi=x^{2 \alpha}$. We consider region $\mathrm{A}$ in which we have the parameterisation $x^{2 c}=-x^{2 b}$ with $-1<b<1$. We find that in the limit $x \rightarrow 1$

$$
\begin{aligned}
& \tilde{M}_{+}^{(1,1)}(\alpha ; r=4, c)=\tilde{M}_{-}^{(2,3)}(\alpha ; r=4,-c) \rightarrow \tan (\pi(\alpha / 2-1 / 4)) \\
& \tilde{M}_{-}^{(2,2)}(\alpha ; r=4, c)=\tilde{M}_{+}^{(1,2)}(\alpha ; r=4,-c) \rightarrow \tan (\pi(\alpha / 2-1 / 4))\left(\frac{\cos (\pi b)+\sin (\pi \alpha)}{\cos (\pi b)-\sin (\pi \alpha)}\right)
\end{aligned}
$$

We now compare these expressions with the boundary S-matrices for the Ising model derived in reference [10]. We find that (6.16) and $(\sqrt[6.17]{)})$ agree with the boundary S-matrices for 'fixed' and 'boundary magnetic field' boundary conditions given in (4.10) and (4.27) of [10] if we identify the rapidity $\theta=\pi i \alpha$, and the magnetic field parameter $\kappa=\cos (\pi b)$. In addition, setting $b=0$ in 6.17) gives the boundary S-matrix for 'free' boundary conditions as given in (4.16) of [10]. 


\subsection{The $r=5$ model}

There are just four independent non-zero S-matrix elements in this case. Their scaling behaviour is given by,

$$
\begin{aligned}
& \tilde{S}\left(\begin{array}{ll|}
1 & 2 \\
2 & 1
\end{array} \mid \alpha\right)=\tilde{S}\left(\begin{array}{ll|l}
3 & 2 \\
2 & 3 & \alpha
\end{array}\right) \rightarrow-\sqrt{2}^{1-\alpha} \cos \left(\frac{\pi}{4}(\alpha-1)\right) F(\alpha)=-B_{0}(\theta), \\
& \tilde{S}\left(\begin{array}{ll}
3 & 2 \\
2 & 1
\end{array} \mid \alpha\right)=\tilde{S}\left(\begin{array}{ll|l}
1 & 2 & \alpha \\
2 & 3 & \alpha
\end{array}\right) \rightarrow-\sqrt{2}^{1-\alpha} \cos \left(\frac{\pi}{4}(\alpha+1)\right) F(\alpha)=-B_{1}(\theta), \\
& \tilde{S}\left(\begin{array}{ll|}
2 & 1 \\
1 & 2
\end{array} \mid \alpha\right)=\tilde{S}\left(\begin{array}{ll|l}
2 & 3 & \alpha \\
3 & 2 & \alpha
\end{array}\right) \rightarrow-\sqrt{2}^{\alpha} \cos \left(\frac{\pi}{4} \alpha\right) F(\alpha) \quad=-A_{0}(\theta), \\
& \tilde{S}\left(\begin{array}{ll|}
2 & 1 \\
3 & 2
\end{array} \mid \alpha\right)=\tilde{S}\left(\begin{array}{ll|l}
2 & 3 & \alpha \\
1 & 2 & \alpha
\end{array}\right) \rightarrow-\sqrt{2}^{\alpha} \sin \left(\frac{\pi}{4} \alpha\right) F(\alpha) \quad=-A_{1}(\theta),
\end{aligned}
$$

where,

$$
F(\alpha)=\prod_{n>0} \frac{\Gamma\left(n+\frac{1}{2}\right)}{\Gamma\left(n-\frac{1}{2}\right)} \frac{\Gamma\left(n-\frac{\alpha}{2}\right) \Gamma\left(n+\frac{\alpha}{2}-\frac{1}{2}\right)}{\Gamma\left(n+\frac{\alpha}{2}\right) \Gamma\left(n-\frac{\alpha}{2}+\frac{1}{2}\right)}=\left[\cos \left(\frac{\pi \alpha}{2}\right)\right]^{-\frac{1}{2}} \exp \left(-\frac{1}{4} \int_{0}^{\infty} \frac{d t}{t} \frac{\sinh (\alpha t)}{\cosh ^{2}(t / 2)}\right) .
$$

The function $F(\alpha)$ has the properties

$$
F(\alpha) F(-\alpha)=1 \text { and } F(1-\alpha)=F(\alpha) .
$$

$B_{0}(\theta), B_{1}(\theta), A_{0}(\theta), A_{1}(\theta)$ are the S-matrix elements given in references [16, 11]. (note however that $F(\alpha)$ is convergent, unlike $S(\theta)$ of references [16, 11]). The representation of $F(\alpha)$ in terms of $\Gamma$ functions was obtained using the limiting behaviour of $\left(p^{z} ; p\right)_{\infty}$ discussed in Section 7.2. The integral representation was constructed by taking the limit of the infinite sum that occurs in $\log \left(p^{z} ; p\right)$, and is equal to the expression given in reference [17]. It is simple to show that the two representations agree by making use of the integral representation of $\log (\Gamma)$.

The symmetry of $M_{\varepsilon}^{(k-i, k)}(\xi ; r, c)=M_{-\varepsilon}^{(r-1-k+i, r-k)}(\xi ; r,-c)$ means that there are four independent boundary S-matrices. They have the following scaling limits:

$$
\begin{aligned}
\tilde{M}_{+}^{(1,1)}(\alpha ; r=5, c)=\tilde{M}_{-}^{(3,4)}(\alpha ; r=5,-c) & \rightarrow \sqrt{2}^{\alpha} \tan \pi\left(\frac{\alpha}{2}-\frac{1}{4}\right) P_{\min }(\alpha), \\
& =e^{\gamma \theta} i \tanh \left(\frac{i \pi}{4}-\frac{\theta}{2}\right) R_{(-1)}(\theta), \\
& =e^{\gamma \theta} i \tanh \left(\frac{i \pi}{4}-\frac{\theta}{2}\right) P_{-}(\theta), \\
\tilde{M}_{-}^{(3,3)}(\alpha ; r=5, c)=\tilde{M}_{+}^{(1,2)}(\alpha ; r=5,-c) & \rightarrow \sqrt{2}^{\alpha} \tan \pi\left(\frac{\alpha}{2}-\frac{1}{4}\right) P_{\min }(\alpha)\left(\frac{\cos (\pi b)+\sin (\pi \alpha)}{\cos (\pi b)-\sin (\pi \alpha)}\right), \\
\tilde{M}_{ \pm}^{(2,2)}(\alpha ; r=5, c)=\tilde{M}_{\mp}^{(2,3)}(\alpha ; r=5,-c) & \rightarrow \\
\sqrt{2}^{-\alpha} \tan \pi\left(\frac{\alpha}{2}-\frac{1}{4}\right) P_{\min }(\alpha)\left(\frac{\cos (\pi b)+\sin (\pi \alpha)}{\cos (\pi b)-\sin (\pi \alpha)}\right) F(\alpha- & \left.-\frac{1}{2}\right) F\left(\alpha+b+\frac{1}{2}\right)\left(\cos \left(\pi\left(\frac{b}{2}+\frac{1}{4}\right) \mp \sin \left(\frac{\pi \alpha}{2}\right)\right),\right. \\
& =e^{\gamma \theta} i \tanh \left(\frac{i \pi}{4}-\frac{\theta}{2}\right) R_{ \pm}(\theta) .
\end{aligned}
$$


where

$$
P_{\text {min }}(\alpha)=\prod_{n>0} \frac{\Gamma^{2}\left(n-\frac{\alpha}{2}\right) \Gamma\left(n+\frac{\alpha}{2}+\frac{1}{4}\right) \Gamma\left(n+\frac{\alpha}{2}-\frac{1}{4}\right)}{\Gamma^{2}\left(n+\frac{\alpha}{2}\right) \Gamma\left(n-\frac{\alpha}{2}+\frac{1}{4}\right) \Gamma\left(n-\frac{\alpha}{2}-\frac{1}{4}\right)}=\exp \left(-2 \int_{0}^{\infty} \frac{d t}{t} \frac{\sinh (\alpha t) \sinh ^{2}(t / 4)}{\sinh ^{2}(t)}\right) .
$$

$P_{\min }(\alpha)$ has the properties

$$
P_{\min }(\alpha) P_{\min }(-\alpha)=1, \quad \text { and } P_{\min }(1-\alpha)=\sqrt{2} \sin (\pi \alpha / 2) F(2 \alpha-1) P_{\min }(\alpha) .
$$

The latter is shown most simply by using the integral representation of both sides. $R_{(-1)}, P_{-}(\theta)$ and $R_{ \pm}(\theta)$ are the boundary S-matrices of reference [11], and $\gamma$ is defined in [11] as $e^{2 \pi i \gamma}=2$ (we identify $\alpha=\frac{\theta}{\pi i}$, and $\pi b=\xi-\frac{\pi}{2}$, where $\xi$ is the magnetic field variable of reference [11]).

We have checked that the scaling limits of our bulk and boundary S-matrices obey the continuum Yang-Baxter, crossing, and unitarity conditions, and we should comment on overall factors by which our results differ from those of references [16, 11]. If we wished, we could remove the overall - sign in our S-matrices by a gauge transformation. However we choose to keep it because it introduces the $\tan \pi(\alpha / 2-1 / 4)$ factor in the boundary S-matrices. As pointed out in reference [11, we expect a simple pole in $M_{-}^{(3,3)}(\alpha ; r, c)=M_{+}^{(1,2)}(\alpha ; r,-c)$ at $\alpha=1 / 2$ when $b=0$. The 'CDD factor' $(\cos (\pi b)+\sin (\pi \alpha)) /(\cos (\pi b)+\sin (\pi \alpha))$ however has a double pole at $\alpha=1 / 2$ when $b=0$. So we need the factor $\tan \pi(\alpha / 2-1 / 4)$ in order to turn the double pole into a simple pole. This situation is just the same as for the $r=4$ Ising model where the same factors are present in $M_{-}^{(2,2)}(\alpha ; r, c)=M+{ }^{(1,2)}(\alpha ; r,-c)$. The absence of the remaining $e^{\gamma \theta}$ in the boundary S-matrix elements of [1] appears to be a simple error. Its presence is necessary to ensure boundary crossing/unitarity.

\section{Discussion}

In summary, we have extended the bosonization scheme of reference [7] to the boundary $\mathrm{ABF}$ models. Specifically, by making use of bozonized vertex operators we have constructed boundary states $|k-i, k\rangle_{B}^{c}$, and the associated boundary S-matrices. We have initially constructed $|k-i, k\rangle_{B}^{c}$ in the bosonic Fock space $\mathcal{F}_{k-i, k}$, and then shown that it determines a non-zero residue class in $\mathcal{L}_{k-i, k}$ under some assumptions on the cohomological construction proposed by Lukyanov and Pugai. For $b>0,|k, k\rangle_{B}^{c}$ is the vacuum state, and $|k-1, k\rangle_{B}^{c} \sim \operatorname{Res}_{\xi=-x^{1-2 c}} \Psi_{-}^{*}(\xi)|k, k\rangle_{B}^{c}$ is interpreted as a bound state of $|k, k\rangle_{B}^{c}$ with a - kink. This bound state is stable for $0<b<\frac{1}{2}$. Conversely, for $b<0,|k-1, k\rangle_{B}^{c}$ is the vacuum state, and $|k, k\rangle_{B}^{c} \sim \operatorname{Res}_{\xi=-x^{1+2 c}} \Psi_{+}^{*}(\xi)|k-1, k\rangle_{B}^{c}$ may be interpreted as a bound state of $|k-1, k\rangle_{B}^{c}$ with a + kink. This bound state is stable for $-\frac{1}{2}<b<0$. General continous eigenstates of the boundary transfer matrix are constructed by acting with the type II vertex operators $\Psi_{\varepsilon}^{*}$ on the discrete states $|k-i, k\rangle_{B}^{c}$.

The scaling limit of our bulk and boundary $S$-matrices should be those associated with the $\phi_{(1,3)}$ perturbation of the $c=1-6 / r(r-1)$ rational conformal field theories. For $r=4$ and $r=5$ we have checked our scaling limit against the results for these perturbed boundary conformal field theories which are available in the literature. 
There are several directions in which this work may be exploited and extended. Using our bosonized expressions for the bounds states and vertex operators, it is possible to write down integral expression for arbitrary equal time correlation functions of local operators of the theory. This may be done by a simple extension of the technique presented in [8]. Integral expressions for form factors may be derived in the same manner. Difference equations for the correlation functions and form factors may be derived following the approach of [18]. The results could be extended to $A_{n-1}^{(1)}$ face models using the bosonization scheme of reference 19.

\section{Acknowledgments}

We wish to thank M. Jimbo, Y. Pugai and P. Dorey for interesting discussions. RAW would like to thank the Royal Society/JSPS/RIMS/Newton Institute exchange scheme for providing him with funds to visit RIMS. He would also like to thank everyone in RIMS for their hospitality during the period in which the initial stages of this work were carried out. 


\section{References}

[1] G.E. Andrews, R.J. Baxter, and P.J Forrester. Eight-vertex SOS Model and Generalized Rogers-Ramanujan-Type Identities. J. Stat. Phys., 35:193-266, 1984.

[2] R. J. Baxter. Exactly Solved Models in Statistical Mechanics. Academic, London, 1982.

[3] B. Davies, O. Foda, M. Jimbo, T. Miwa, and A. Nakayashiki. Diagonalisation of the XXZ Hamiltonian by vertex operators. Comm. Math. Phys., 151:89-153, 1993.

[4] M. Jimbo and T. Miwa. Algebraic Analysis of Solvable Lattice Models. CBMS Regional Conference Series in Mathematics vol. 85, AMS, 1994.

[5] A. Tsuchiya and Y. Kanie. Vertex operators in conformal field theory on $\mathbf{P}^{1}$ and representations of the braid group. Adv. Stud. in Pure Math., 16:297-372, 1988.

[6] B. L. Feigin and D. B. Fuchs. Representations of the Virasoro Algebra, 1984. In: Topology, Proceedings, Leningrad 1982, Ed. by L.D. Faddeev and A.A. Mal'cev. Lecture Notes in Mathematics, Vol. 1060, Springer.

[7] S. Lukyanov and Y. Pugai. Multi-point Local Height Probabilities in the Integrable RSOS Models. Nucl. Phys., B473:631-658, 1996.

[8] M. Jimbo, R. Kedem, T. Kojima, H. Konno, and T. Miwa. XXZ chain with a boundary. Nucl. Phys., B441 [FS]:437-470, 1995.

[9] R.E. Behrend, P.A. Pearce, and D.L. O'Brien. Interaction-Round-a-Face Models with Fixed Boundary Conditions: The ABF Fusion Hierarchy. J. Stat. Phys., 84:84, 1996.

[10] S. Ghoshal and A. Zamolodchikov. Boundary $S$-matrix and boundary state in two-dimensional integrable quantum field theory. Int. J. Mod. Phys., A9:3841-3885, 1994.

[11] L. Chim. Boundary S-matrix for the Tricritical Ising Model. Int. J. Mod. Phys., A11:44914512, 1996.

[12] E. K. Sklyanin. Boundary conditions for integrable quantum systems. J. Phys. A, 21:2375$2389,1988$.

[13] M. Jimbo, M. Lashkevich, T. Miwa, and L. Pugai. Lukyanov's Screening Operators for the Deformed Virasoro Algebra, 1996. Preprint hep-th/9607177.

[14] S. Ghoshal. Bound state boundary S-matrix of the sine-gordan model. Int. J. Mod. Phys., A9:4801-4810, 1994.

[15] D. Bernard and A LeClair. Residual Quantum Symmetries of the Restricted Sine-Gordan Theories. Nucl. Phys., B340:721-751, 1990.

[16] A.B. Zamolodchikov. Fractional-spin Integrals of Motion in Perturbed Conformal Field Theory. Published in Proc. of 'Field Strings an Quantum Gravity' - Beijing 1989 (Gordan and Breach), Ed. H. Guo, A. Qiu and H. Tye.

[17] Al. B. Zamolodchikov. Thermodynamic Bethe Ansatz for RSOS Scattering Theories. Nucl. Phys., B358:497-523, 1991.

[18] M. Jimbo, R. Kedem, H. Konno, T. Miwa, and R.A. Weston. Difference equations in spin chains with a boundary. Nucl. Phys., B448:429-456, 1995.

[19] Y. Asai, M. Jimbo, T. Miwa, and Y. Pugai. Bosonization of vertex operators for the $A_{n-1}^{(1)}$ face model. Preprint hep-th/9606095. 


\section{A Normal ordering relations and summation identities}

\section{A.1 Normal ordering relations for vertex operators}

We list the normal ordering relations for the vertex operators discussed in Section 3. We abbreviate $A B=: A B: C$ to $A B=:: C$, and use $\zeta=x^{2 u}$ and $z=x^{2 v}$.

$$
\begin{aligned}
& \Phi_{+}\left(\zeta_{1}\right) \Phi_{+}\left(\zeta_{2}\right)=:: \zeta_{1}^{-\left(\frac{r-1}{2 r}\right)} \frac{\left(x^{2} \zeta_{1} / \zeta_{2} ; x^{4}, x^{2 r}\right)_{\infty}\left(x^{2+2 r} \zeta_{1} / \zeta_{2} ; x^{4}, x^{2 r}\right)_{\infty}}{\left(x^{2 r} \zeta_{1} / \zeta_{2} ; x^{4}, x^{2 r}\right)_{\infty}\left(x^{4} \zeta_{1} / \zeta_{2} ; x^{4}, x^{2 r}\right)_{\infty}}, \\
& \Phi_{+}(\zeta) A(z)=: \quad: \zeta^{\left(\frac{r-1}{r}\right)} \frac{\left(z \zeta x^{2 r-1} ; x^{2 r}\right)_{\infty}}{\left(z \zeta x ; x^{2 r}\right)_{\infty}}, \\
& A(z) \Phi_{+}(\zeta)=: \quad: z^{-\left(\frac{r-1}{r}\right)} \frac{\left((z \zeta)^{-1} x^{2 r-1} ; x^{2 r}\right)_{\infty}}{\left((z \zeta)^{-1} x ; x^{2 r}\right)_{\infty}}, \\
& \Phi_{+}(\zeta) A(z)=A(z) \Phi_{+}(\zeta) \frac{\left[\frac{1}{2}-u-v\right]}{\left[\frac{1}{2}+u+v\right]}, \\
& A\left(z_{1}\right) A\left(z_{2}\right)=: \quad: z_{1}^{\frac{2(r-1)}{r}}\left(1-z_{2} / z_{1}\right) \frac{\left(x^{2} z_{2} / z_{1} ; x^{2 r}\right)_{\infty}}{\left(x^{2(r-1)} z_{2} / z_{1} ; x^{2 r}\right)_{\infty}}, \\
& A\left(z_{1}\right) A\left(z_{2}\right)=\frac{\left[v_{1}-v_{2}-1\right]}{\left[v_{1}-v_{2}+1\right]} A\left(z_{2}\right) A\left(z_{1}\right), \\
& \Psi_{+}^{*}\left(\zeta_{1}\right) \Psi_{+}^{*}\left(\zeta_{2}\right)=: \quad: \zeta_{1}^{-\left(\frac{r}{2(r-1)}\right)} \frac{\left(\zeta_{1} / \zeta_{2} ; x^{4}, x^{2(r-1)}\right)_{\infty}\left(x^{2(r+1)} \zeta_{1} / \zeta_{2} ; x^{4}, x^{2(r-1)}\right)_{\infty}}{\left(x^{2 r} \zeta_{1} / \zeta_{2} ; x^{4}, x^{2(r-1)}\right)_{\infty}\left(x^{2} \zeta_{1} / \zeta_{2} ; x^{4}, x^{2(r-1)}\right)_{\infty}}, \\
& \Psi_{+}^{*}(\zeta) B(z)=:: \zeta^{\left(\frac{r}{r-1}\right)} \frac{\left(z \zeta x^{2 r-1} ; x^{2(r-1)}\right)_{\infty}}{\left(z \zeta x^{-1} ; x^{2(r-1)}\right)_{\infty}},
\end{aligned}
$$

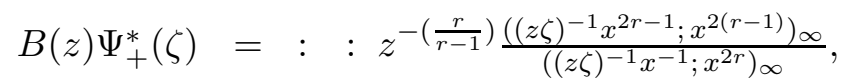

$$
\begin{aligned}
& B(z) \Psi_{+}^{*}(\zeta)=\Psi_{+}^{*}(\zeta) V(z) \frac{\left[\frac{1}{2}-u-v\right]^{\prime}}{\left[\frac{1}{2}+u+v\right]^{\prime}}, \\
& \Phi_{+}\left(\zeta_{1}\right) \Psi_{+}^{*}\left(\zeta_{2}\right)=:: \zeta_{1}^{\frac{1}{2}} \frac{\left(-x^{3} \zeta_{1} / \zeta_{2} ; x^{4}\right)_{\infty}}{\left(-x \zeta_{1} / \zeta_{2} ; x^{4}\right)_{\infty}}, \\
& \Psi_{+}^{*}\left(\zeta_{1}\right) \Phi_{+}\left(\zeta_{2}\right)=:: \zeta_{1}^{\frac{1}{2}} \frac{\left(-x^{3} \zeta_{1} / \zeta_{2} ; x^{4}\right)_{\infty}}{\left(-x \zeta_{1} / \zeta_{2} ; x^{4}\right)_{\infty}}, \\
& \Phi_{+}\left(\zeta_{1}\right) \Psi_{+}^{*}\left(\zeta_{2}\right)=\left(\zeta_{1} / \zeta_{2}\right)^{\frac{1}{2}} \frac{\theta_{x^{4}}\left(-x \zeta_{2} / \zeta_{1}\right)}{\theta_{x^{4}}\left(-x \zeta_{1} / \zeta_{2}\right)} \Psi_{+}^{*}\left(\zeta_{2}\right) \Phi_{+}\left(\zeta_{1}\right), \\
& \Phi_{+}(\zeta) B(z)=:: \zeta^{-1}(1+\zeta z), \\
& B(z) \Phi_{+}(\zeta)=:: z\left(1+(\zeta z)^{-1}\right), \\
& \Phi_{+}(\zeta) B(z)=B(z) \Phi_{+}(\zeta) \\
& \Psi_{+}^{*}(\zeta) A(z)=:: \zeta^{-1}(1+\zeta z), \\
& A(z) \Psi_{+}^{*}(\zeta)=:: z\left(1+(\zeta z)^{-1}\right), \\
& \Psi_{+}^{*}(\zeta) A(z)=A(z) \Psi_{+}^{*}(\zeta), \\
& A\left(z_{1}\right) B\left(\zeta_{2}\right)=: \quad: \frac{1}{z_{1}^{2}\left(1+x z_{2} / z_{1}\right)\left(1+x^{-1} z_{2} / z_{1}\right)}, \\
& B\left(z_{2}\right) A\left(\zeta_{1}\right)=:: \frac{1}{z_{2}^{2}\left(1+x z_{1} / z_{2}\right)\left(1+x^{-1} z_{1} / z_{2} 1\right)}, \\
& A\left(z_{1}\right) B\left(\zeta_{2}\right)=B\left(z_{2}\right) A\left(\zeta_{1}\right), \\
& B\left(z_{1}\right) B\left(z_{2}\right)=: \quad: z_{1}^{\frac{2 r}{r-1}}\left(1-z_{2} / z_{1}\right) \frac{\left(x^{-2} z_{2} / z_{1} ; x^{2(r-1)}\right)_{\infty}}{\left(x^{2 r} z_{2} / z_{1} ; x^{2(r-1)}\right)_{\infty}}, \\
& B\left(z_{1}\right) B\left(z_{2}\right)=\frac{\left[v_{1}-v_{2}+1\right]^{\prime}}{\left[v_{1}-v_{2}-1\right]^{\prime}} B\left(z_{2}\right) B\left(z_{1}\right) \text {. }
\end{aligned}
$$




\section{A.2 Summation identities}

$$
\begin{aligned}
e^{\sum_{m>0} \frac{z^{m}}{m} \frac{[m] x[r m] x}{[2 m]_{x}[(r-1) m]_{x}}} & =\frac{\left(x^{2 r} z ; x^{4}, x^{2(r-1)}\right)_{\infty}\left(x^{2} z ; x^{4}, x^{2(r-1)}\right)_{\infty}}{\left(z ; x^{4}, x^{2(r-1)}\right)_{\infty}\left(x^{2(1+r)} z ; x^{4}, x^{2(r-1)}\right)_{\infty}}, \\
e^{m>0} \frac{z^{m}}{m} \frac{[m] x[(r-1) m] x}{[2 m] x[r m]_{x}} & =\frac{\left(x^{2 r} z ; x^{4}, x^{2 r}\right)_{\infty}\left(x^{4} z ; x^{4}, x^{2 r}\right)_{\infty}}{\left(x^{2} z ; x^{4}, x^{2 r}\right)_{\infty}\left(x^{2(1+r)} z ; x^{4}, x^{2 r}\right)_{\infty}}, \\
\sum^{\sum^{m>0} \frac{z^{m}}{m} \frac{[m] x}{[2 m]_{x}}} & =\frac{\left(x^{3} z ; x^{4}\right)_{\infty}}{\left(x z ; x^{4}\right)_{\infty}} \\
\sum^{m>0} \frac{z^{m}}{m} \frac{[(r-1) m] x}{[r m]_{x}} & =\frac{\left(x^{2 r-1} z ; x^{2 r}\right)_{\infty}}{\left(x z ; x^{2 r}\right)_{\infty}} \\
\sum^{m>0} \frac{z^{m}}{m} \frac{[r m]_{x}}{[(r-1) m]_{x}} & =\frac{\left(x^{2 r-1} z ; x^{2(r-1)}\right)_{\infty}}{\left(x^{-1} z ; x^{2(r-1)}\right)_{\infty}} .
\end{aligned}
$$

\section{B Proof of (4.6) and (4.7) for $\varepsilon=-$ and general $k$}

In this appendix we shall give an inductive proof of (4.6) and (4.7) for $\varepsilon=-$ and general $k$. The first step is to show that if we have

$$
K_{>}^{(c)}\left(k \pm 1_{k}^{k} \mid u\right) \Phi_{ \pm}(\zeta)|k, k\rangle_{B}^{c}=\Phi_{ \pm}\left(\zeta^{-1}\right)|k, k\rangle_{B}^{c}
$$

for a given value of $k$, then it follows that

$$
K_{<}^{(c)}\left(k_{k+1}^{k+1} \mid u\right) \Phi_{-}(\zeta)|k, k+1\rangle_{B}^{c}=\Phi_{-}\left(\zeta^{-1}\right)|k, k+1\rangle_{B}^{c}
$$

is also true for the same value of $k$. Using (4.29) and the vertex operator commutation relations (3.49), we can rewrite (B.2) as

$$
\begin{aligned}
& K_{<}^{(c)}\left(k_{k+1}^{k+1} \mid u\right)\left\{W\left(\begin{array}{ll}
k & k+1 \\
k+1 & k
\end{array} \mid c+1-u\right) \Phi_{-}\left(x^{2 c+2}\right) \Phi_{+}(\zeta)\right. \\
& \left.+W\left(\begin{array}{ll|}
k & k-1 \\
k+1 & k
\end{array} \mid c+1-u\right) \Phi_{+}\left(x^{2 c+2}\right) \Phi_{-}(\zeta)\right\}|k, k\rangle_{B}^{c+1}= \\
& \left\{W\left(\begin{array}{ll}
k & k+1 \\
k+1 & k
\end{array} \mid c+1+u\right) \Phi_{-}\left(x^{2 c+2}\right) \Phi_{+}\left(\zeta^{-1}\right)\right. \\
& +W\left(\begin{array}{ll}
k & k-1 \\
k+1 & k
\end{array}\right.
\end{aligned}
$$

We can now use the identities

$$
\begin{aligned}
& K_{<}^{(c)}\left(\begin{array}{l}
k+1 \\
k+1
\end{array} \mid u\right) W\left(\begin{array}{ll}
k & k+1 \\
k+1 & k
\end{array} \mid c+1-u\right)=K_{>}^{(c+1)}\left(k+1 \frac{k}{k} \mid u\right) W\left(\begin{array}{ll}
k & k+1 \\
k+1 & k
\end{array} \mid c+1+u\right), \\
& K_{<}^{(c)}\left(\begin{array}{l}
k+1 \\
k+1
\end{array} \mid u\right) W\left(\begin{array}{ll}
k & k-1 \\
k+1 & k
\end{array} \mid c+1-u\right)=K_{>}^{(c+1)}\left(k-1 \frac{k}{k} \mid u\right) W\left(\begin{array}{ll}
k & k-1 \\
k+1 & k
\end{array} \mid c+1+u\right),
\end{aligned}
$$

and the given relations (B.1) to re-write the LHS of (B.3) such that it is identically equal to the RHS. 
The next step in the proof is show that if

$$
K_{<}^{(c)}\left(k-1_{k}^{k} \mid u\right) \Phi_{-}(\zeta)|k-1, k\rangle_{B}^{c}=\Phi_{-}\left(\zeta^{-1}\right)|k-1, k\rangle_{B}^{c}
$$

is true for a given $k$, then

$$
K_{>}^{(c)}\left(k-1_{k}^{k} \mid u\right) \Phi_{-}(\zeta)|k, k\rangle_{B}^{c}=\Phi_{-}\left(\zeta^{-1}\right)|k, k\rangle_{B}^{c}
$$

follows. To show this we rewrite (B.5), using (4.30) and the commutation relations (3.54), as

$$
\begin{aligned}
& \operatorname{Res}_{\xi=-x^{1+2 c}} \chi(\xi / \zeta) K_{>}^{(c)}\left(k-1_{k}^{k} \mid u\right) \Psi_{+}^{*}(\xi) \Phi_{-}(\zeta)|k-1, k\rangle_{B}^{c} \\
& =\operatorname{Res}_{\xi=-x^{1+2 c}} \chi(\xi \zeta) \Psi_{+}^{*}(\xi) \Phi_{-}\left(\zeta^{-1}\right)|k-1, k\rangle_{B}^{c} .
\end{aligned}
$$

The equality of the two sides is then established using (B.5) and the identity

$$
\chi(\xi / \zeta) K_{>}^{(c)}\left(k-1_{k}^{k} \mid u\right)=\chi(\xi \zeta) K_{<}^{(c)}\left(k-1_{k}^{k} \mid u\right) .
$$

We have now completed the inductive steps. In Section 4 we proved (4.6) for $\varepsilon=-$ and $k=1$ (4.6) with $\varepsilon=+$ and general $k$ is true by construction). The proof of (4.6) and (4.7) is complete. 